\title{
A General Embedding Framework for Heterogeneous Information Learning in Large-Scale Networks
}

\author{
XIAO HUANG, Texas A\&M University \\ JUNDONG LI, Arizona State University \\ NA ZOU and XIA HU, Texas A\&M University
}

\begin{abstract}
Network analysis has been widely applied in many real-world tasks, such as gene analysis and targeted marketing. To extract effective features for these analysis tasks, network embedding automatically learns a low-dimensional vector representation for each node, such that the meaningful topological proximity is well preserved. While the embedding algorithms on pure topological structure have attracted considerable attention, in practice, nodes are often abundantly accompanied with other types of meaningful information, such as node attributes, second-order proximity, and link directionality. A general framework for incorporating the heterogeneous information into network embedding could be potentially helpful in learning better vector representations. However, it remains a challenging task to jointly embed the geometrical structure and a distinct type of information due to the heterogeneity. In addition, the real-world networks often contain a large number of nodes, which put demands on the scalability of the embedding algorithms. To bridge the gap, in this article, we propose a general embedding framework named Heterogeneous Information Learning in Large-scale networks (HILL) to accelerate the joint learning. It enables the simultaneous node proximity assessing process to be done in a distributed manner by decomposing the complex modeling and optimization into many simple and independent sub-problems. We validate the significant correlation between the heterogeneous information and topological structure, and illustrate the generalizability of HILL by applying it to perform attributed network embedding and second-order proximity learning. A variation is proposed for link directionality modeling. Experimental results on real-world networks demonstrate the effectiveness and efficiency of HILL.
\end{abstract}

CCS Concepts: - Information systems $\rightarrow$ Information integration; Web mining; • Computing methodologies $\rightarrow$ Distributed computing methodologies;

Additional Key Words and Phrases: Data mining, network embedding, heterogeneity, distributed processing

ACM Reference format:

Xiao Huang, Jundong Li, Na Zou, and Xia Hu. 2018. A General Embedding Framework for Heterogeneous Information Learning in Large-Scale Networks. ACM Trans. Knowl. Discov. Data 12, 6, Article 70 (October 2018), 24 pages.

https://doi.org/10.1145/3241063

This work is, in part, supported by DARPA (\#N66001-17-2-4031) and NSF (\#IIS-1657196 and \#IIS-1718840). The views, opinions, and/or findings expressed are those of the author(s) and should not be interpreted as representing the official views or policies of the Department of Defense or the U.S. Government.

Authors' addresses: X. Huang, N. Zou (Corresponding author), and X. Hu, Texas A\&M University, College Station, TX 77843; emails: \{xhuang, nzou1, xiahu\}@tamu.edu; J. Li, Arizona State University, Tempe, AZ 85281; email: jundongl@asu.edu.

Permission to make digital or hard copies of all or part of this work for personal or classroom use is granted without fee provided that copies are not made or distributed for profit or commercial advantage and that copies bear this notice and the full citation on the first page. Copyrights for components of this work owned by others than ACM must be honored. Abstracting with credit is permitted. To copy otherwise, or republish, to post on servers or to redistribute to lists, requires prior specific permission and/or a fee. Request permissions from permissions@acm.org.

(C) 2018 Association for Computing Machinery.

1556-4681/2018/10-ART70 \$15.00

https://doi.org/10.1145/3241063 


\section{INTRODUCTION}

Network analysis has become an effective computational tool in various real-world information systems. For example, in social media, service providers recommend potential friendships to users with link prediction techniques [2]. In proteomics, a surge of research is to identify the protein functions, and node classification [48] has been found to be significantly instructive. However, to perform these analysis tasks, identifying effective features often involves huge amounts of human efforts and massive engineering experimentations. As an alternative, network embedding $[44,52]$ automatically maps the topological structure of each node into a low-dimensional vector representation, such that the original network proximity could be well preserved. The learned continuous low-dimensional representations have been demonstrated to be helpful to various analysis tasks, such as node classification [42, 58], link prediction [9, 20], network clustering [35, 51], community detection [53], and anomaly detection [32].

While most of the existing network embedding algorithms focus on pure topological structure $[11,36]$, nodes in real-world systems are often associated with other types of meaningful information. We named this distinct type of information other than topological structure as the heterogeneous information, with examples from node attributes, second-order proximity, to link directionality. First, in attributed networks [18], not only pairwise dependencies are available, but also a rich set of data describing the characteristic attributes and properties of each node is observed. Second, in practice, the direct node-to-node tie strengths might not be enough to capture all the sophisticated underlying patterns, and the second-order proximity [44] could be a complement, which describes the number of intersections in every two nodes' neighborhoods. Third, in directed networks such as the Twitter follower network and the Epinions trust network, the link directionality plays a crucial role in determining the network properties.

The heterogeneous information is usually highly correlated with the network structure. For instance, social science theories such as homophily hypothesis $[33,34]$ and social influence $[49,57]$ suggest that the network structure and node attributes tend to be mutually dependent on each other, i.e., the formation of one depends on and also influences the other. Figure 1 illustrates a toy attributed network of Twitter. The links capture the interactions among users, and network embedding aims to map them into continuous vector representations, which could be applied to different applications. Meanwhile, a user like Texas A\&M University also posts many tweets and comments that reflect her attributes. These posts have strong associations with her following relationships. Another example is the high correlation of paper topics and citations in academic networks [56]. In addition, in various applications such as sentiment analysis [16] and trust prediction [17, 43], it has been shown that jointly exploiting the two information sources could enhance the learning performance. Motivated by these observations, we explore to design a general framework for incorporating the heterogeneous information into network embedding, which is a crucial problem, since it is potentially helpful in learning more informative vector representations.

On the other hand, real-world networks are often large-scale with a large number of nodes and a high-dimensional heterogeneous information [18, 37]. For instance, there are over 65 million monthly active Twitter users in the United States as of $2016,{ }^{1}$ and each user could post up to thousands of tweets, known as node attributes. The second-order proximity [44] includes the affinity information of every pair of nodes' neighborhoods. It increases exponentially as the number of node increases. These all put demands on the scalability of the joint embedding framework.

In summary, it remains a challenging task to collectively embed the network structure and the heterogeneous information, with three major challenges to be addressed. First, high

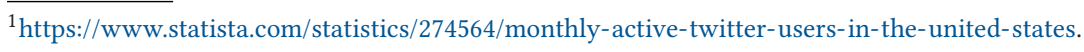




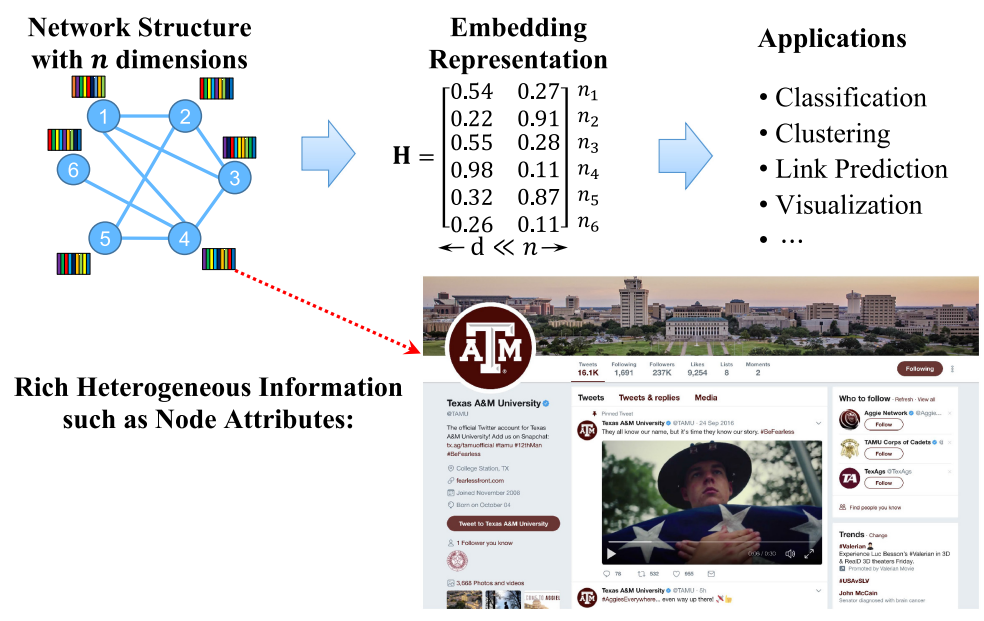

Fig. 1. Network embedding is widely used in many real-world applications, while in an attributed network, a node like Texas A\&M University is often associated with rich data reflecting its characteristics.

computational time requirement could be a bottleneck that limits the application of the joint framework in practice. Some efforts [27, 41] have been devoted to leveraging the network structure and node attributes for seeking a joint low-rank latent representation. They either require eigen-decomposition $[18,26]$ with $O\left(n^{3}\right)$ time complexity in each iteration ( $n$ denotes the total number of nodes) or employ gradient descent $[41,54,58]$ which usually has a slow convergence rate. Hence, it would be appealing to have a general and scalable framework. Second, assessing a vector representation for each node in the joint space of geometrical structure and a distinct type of information is difficult due to the bewildering combination of heterogeneous sources. A traditional solution to combine heterogeneous information sources is to learn from their corresponding affinity matrices $[18,26]$. However, as the size of network scales up, these affinity matrices could become too large to be cached on a single machine, not to mention the operations on them. Third, both of the topological structure and the heterogeneous information could be incomplete and noisy due to the imperfect data collection, which further exacerbates the joint embedding representation learning problem. Therefore, given the distinct characteristics of the data, existing methods cannot be directly applied to incorporate the heterogeneous information into the network embedding.

To tackle the above challenges, in this article, we study the problem of heterogeneous information learning with joint network embedding. It could be summarized into three major research questions as follows. (1) How to effectively model node proximity in the unified space composed of both network structure and the heterogeneous information? (2) How to enable the joint vector representation learning process scalable and efficient? (3) What is the impact of the heterogeneous information learning on network embedding and different real-world applications? Through investigating these questions, we present a general embedding framework named Heterogeneous Information Learning in Large-scale networks (HILL) to accelerate the joint learning. The major contributions of this article could be summarized as follows:

-Empirically validate the correlation between the network structure and the heterogeneous information proximity on real-world datasets;

- Propose a general, effective, and scalable embedding framework HILL to learn a lowdimensional representation simultaneously from the heterogeneous information affinity matrix and topological structure information; 
- Present a distributed algorithm to accelerate HILL by decomposing the complex modeling and optimization into many independent sub-problems of low complexity;

- Illustrate the generalizability of HILL with applications to attributed network embedding, second-order proximity learning, and link directionality modeling, and demonstrate the effectiveness and efficiency of HILL on three real-world datasets.

The remainder of this article is organized as follows. In Section 2, we introduce the notations used and formally define the problem of heterogeneous information learning with joint network embedding. In Section 3, we employ node attribute information as an example of the heterogeneous information, and corroborate its correlation with topological structure on real-world datasets. In Section 4, we propose a general embedding framework HILL, which could incorporate the proximity of the heterogeneous information into the network embedding. In Section 5, we apply HILL to attributed network embedding, second-order proximity learning, and link directionality modeling. In Section 6, we present a distributed optimization algorithm to accelerate HILL. In Section 7, we report and evaluate the empirical results on three real-world networks. In Section 8, we review the existing literature related to our work. In Section 9, we conclude and present the future work.

\section{PROBLEM STATEMENT}

Notations: In this article, scalars are denoted by lowercase alphabets (e.g., $n$ ). Vectors are represented by boldface lowercase alphabets (e.g., h). Matrices are denoted by boldface uppercase alphabets (e.g., $\mathbf{H})$. The $i$ th row of a matrix $\mathbf{H}$ is represented by $\mathbf{h}_{i}$. The element in the $i$ th row and $j$ th column of a matrix is denoted by $h_{i j}$. The pseudoinverse of a matrix $\mathbf{H}$ is represented by $\mathbf{H}^{\dagger}$. The transpose of $\mathbf{H}$ is represented by $\mathbf{H}^{\top}$. The identity matrix is denoted by $\mathrm{I}$. The $\ell_{2}$-norm of a vector is represented by $\|\cdot\|_{2}$, e.g., $\|\mathbf{h}\|_{2}=\sqrt{\sum_{i=1}^{n} h_{i}^{2}}$. The Frobenius norm of a matrix is denoted by $\|\cdot\|_{\mathrm{F}}$.

Definition 1 (Heterogeneous Information). In real-world information networks, there is often another type of meaningful information other than topological structure, such as node attributes, second-order proximity, and link directionality. We refer this distinct data as the heterogeneous information.

Definition 2 (Node Attributes). In a network, we refer the extra textual, numerical, or image data that describes the characteristic features, records, or properties of each node as node attributes. Examples include posts and comments in social media, paper abstracts in academic networks, and property descriptions in protein-protein interaction networks.

Definition 3 (Second-order Proximity). In a network, two nodes might have common neighborhoods with different strengths of relationships. The second-order proximity refers to the affinity of every two nodes' neighborhoods and corresponding relationships.

We list the main symbols in this article in Table 1 . Let $\mathcal{G}=(\mathcal{V}, \mathcal{E}, \mathrm{W})$ be a network, where $\mathcal{V}$ is a set of $n$ nodes, and $\mathcal{E}$ is the corresponding set of edges. Each edge $(i, j) \in \mathcal{E}$ is associated with a positive weight $w_{i j} \in \mathbf{W}$. A larger $w_{i j}$ indicates a higher similarity or stronger relationship between nodes $i$ and $j$, and $w_{i j}$ is defined as 0 if there is no edge. The set of adjacent nodes of node $i$ is represented by $N(i)$. In this article, we focus on undirected networks first, and then present detailed solutions to handle directed networks with or without node attributes. Let A be an $n \times m$ matrix that collects the heterogeneous information of all nodes, where $m$ is the number of heterogeneous information categories, and row $\mathbf{a}_{i}$ describes the heterogeneous information associated with node $i$.

Based on the terminologies explained above, we formally define the problem of heterogeneous information learning with joint network embedding as follows. Given a large set of $n$ nodes 
Table 1. The Main Notations and Definitions in this Article

\begin{tabular}{ccc}
\hline Notations & Space & Definitions \\
\hline$n=|\mathcal{V}|$ & $\mathbb{R}$ & The number of nodes in the network \\
$m$ & $\mathbb{R}$ & The number of heterogeneous information categories \\
$d$ & $\mathbb{R}$ & The dimension of embedding representation \\
$N(i)$ & Non-constant & The set of adjacent nodes of node $i$ \\
$\mathrm{~W}$ & $\mathbb{R}_{+}^{n \times n}$ & The weighted adjacency matrix \\
$\mathrm{A}$ & $\mathbb{R}^{n \times m}$ & The heterogeneous information matrix \\
$\mathrm{S}$ & $\mathbb{R}^{n \times n}$ & The heterogeneous information affinity matrix \\
$\mathrm{H}$ & $\mathbb{R}^{n \times d}$ & The final embedding representation \\
$\mathrm{Z}$ & $\mathbb{R}^{n \times d}$ & A copy of $\mathrm{H}$ for optimization \\
\hline
\end{tabular}

connected by a network $\mathcal{G}=(\mathcal{V}, \mathcal{E}, \mathrm{W})$ associated with a high-dimensional heterogeneous information $\mathbf{A}$, we aim to represent each node $i \in \mathcal{V}$ as a d-dimensional vector $\mathbf{h}_{i}$, such that the entire embedding representation $\mathrm{H}$ can preserve all the node proximity in both topological structure $\mathrm{W}$ and heterogeneous information A. As a result, $\mathrm{H}$ could achieve better performance than pure network embedding in terms of advancing other learning tasks such as node classification.

\section{DATA ANALYSIS ON REAL-WORLD NETWORKS}

In this section, we take node attribute information as an example of heterogeneous information, and verify its correlation with the network typological structure on the real-world datasets. Three real-world attributed networks, i.e., BlogCatalog, Flickr, and Yelp are used in this work. All of them are publicly available and have been used in several previous work $[18,29]$. The statistics of the three datasets are summarized in Table 2, with detailed descriptions as follows.

BlogCatalog is a blogger community, where users interact with each other and form a network. Users are allowed to generate keywords as a short description of their blogs. These keywords are served as node attributes. Users also register their blogs under predefined categories, and we set them as labels. The user with no follower or predefined category has been removed.

Flickr is an online community that people could share photos. These photographers could follow each other and form a network. We employ the tags specified on their images as the node attribute information. We set the groups that photographers joined as labels.

Yelp ${ }^{2}$ is a social networking service, where crowd-sourced reviews about local businesses are shared. We employ users' friend relationships to form the network, and the bag-of-words model to represent users' reviews as node attributes. All local businesses are separated into 11 primary categories, including Active Life, Arts \& Entertainment, Food, Hotels \& Travel, Nightlife, American Restaurants, Non-American Restaurants, Fast Food \& Meat, Other Restaurants, Shopping, and Services. A user may have reviewed one or several businesses. We use the categories of these businesses as the user's labels.

The well-received studies such as homophily and social influences [34, 57] suggest that nodes with similar network structure would tend to have similar node attributes. We now verify this correlation experimentally. Since there is no formal definition for matrix correlation, we employ three types of metrics in the validation as follows.

Correlation Metrics: The first metric CorrCoef is defined as the Pearson correlation coefficient of all pairwise affinities in the network and node attribute space. There are $\left(\begin{array}{l}n \\ 2\end{array}\right)$ node pairs in total,

\footnotetext{
${ }^{2}$ https://www.yelp.com/dataset_challenge/dataset.
} 
Table 2. The Detailed Information of the Three Real-World Attributed Networks

\begin{tabular}{cccccc}
\hline Dataset & Nodes $(n)$ & Edges $(|\mathcal{E}|)$ & Density & Attribute Categories $(m)$ & Label $(\ell)$ \\
\hline BlogCatalog & 5,196 & 171,743 & $1.3 \mathrm{e}-002$ & 8,189 & 6 \\
Flickr & 7,564 & 239,365 & $8.4 \mathrm{e}-003$ & 12,047 & 9 \\
Yelp & 249,012 & $1,779,803$ & $5.7 \mathrm{e}-005$ & 20,000 & 11 \\
\hline
\end{tabular}

Table 3. Correlation Between the Network Proximity and Node

Attribute Proximity w.r.t. the Three Metrics, with a Significance

Level of 0.05

\begin{tabular}{ccccc}
\hline Dataset & Scenarios & CorrCoef & Intersect & p-value \\
\hline \multirow{3}{*}{ BlogCatalog } & Real-world & $3.69 e-002$ & 42 & $\mathbf{0 . 0 0 e - 0 1 6}$ \\
& RandomMean & $3.14 \mathrm{e}-005$ & 7.32 & 0.18 \\
& RandomMax & $1.40 \mathrm{e}-003$ & 13 & $4.42 \mathrm{e}-016$ \\
\hline \multirow{3}{*}{ Flickr } & Real-world & $\mathbf{1 . 8 5 e - 0 0 2}$ & 25 & $\mathbf{0 . 0 0 e - 0 1 6}$ \\
& RandomMean & $2.15 \mathrm{e}-005$ & 3.56 & 0.49 \\
& RandomMax & $5.48 \mathrm{e}-004$ & 9 & $3.37 \mathrm{e}-003$ \\
\hline
\end{tabular}

and we first calculate their cosine similarities based on network structure $\mathbf{W}$ and set them as the

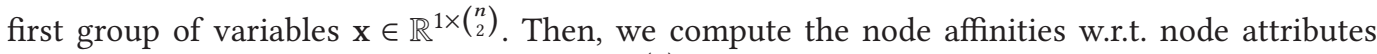

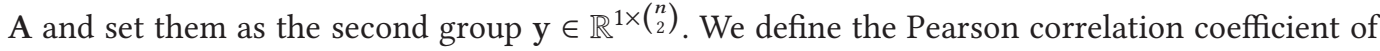
pairwise affinities $\mathbf{x}$ and $\mathbf{y}$ as CorrCoef. The second metric Intersect is defined as the number of common node pairs in the top $10^{4}$ largest affinities in $\mathbf{x}$ and the top $10^{4}$ largest affinities in $\mathbf{y}$. The third metric $p$-value is defined as the p-value of the null hypothesis as follows.

Hyротнеsis 1. There is no correlation between network affinities $\mathbf{x}$ and node attribute affinities $\mathbf{y}$.

To validate the correlation between the topological structure and node attributes, we include a randomly generated network as a baseline. This network has the same density as the real-world network, with undirected edges distributed randomly. We measure the correlation between the randomly generated network and real-world node attributes, and conduct 100 trials for each dataset. We define the average mean as RandomMean and the best performance as RandomMax. The results on BlogCatalog and Flickr are shown in Table 3. The result on Yelp is not available, since it has too many pairwise affinities to be cached on a single machine. As we can see, on both datasets, CorrCoef and Intersect of the real-world networks are much larger than the ones of the randomly generated networks. The p-value of real-world network is $0.00 \mathrm{e}-016$ on both datasets, which demonstrates that there is a significant relationship between the network proximity and node attribute proximity.

\section{A GENERAL EMBEDDING FRAMEWORK - HILL}

To investigate the problem of heterogeneous information learning with joint network embedding, we propose a general, effective, and distributed framework named HILL. In this section, we describe how HILL jointly models the topological structure and heterogeneous information proximity in an efficient way. HILL satisfies three nice properties as follows. First, it is capable of handling various types of edges (e.g., undirected or directed, unweighted or weighted) and an arbitrary type of heterogeneous information (e.g., node attributes or second-order proximity). Second, it could well preserve the node proximity in both network and heterogeneous information space. Third, it 


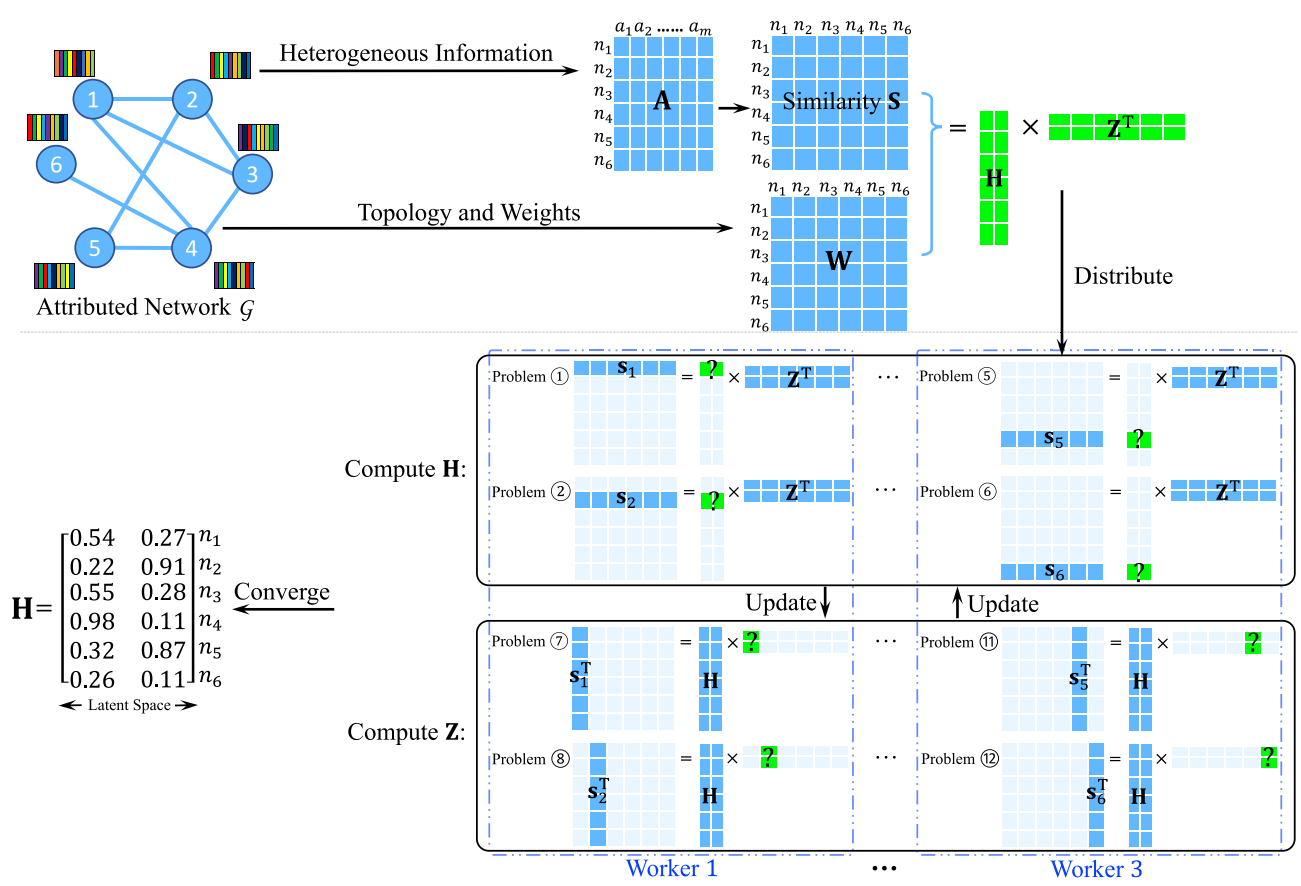

Fig. 2. The key idea of HILL is to represent nodes as continuous vectors, based on the decomposition of heterogeneous information affinity matrix and penalty of embedding difference between adjacent nodes. The optimization is split into $2 n$ sub-problems of low complexity, which can be solved separately by $c$ workers.

is scalable, which is important since the number of nodes $n$ and the dimension of heterogeneous information $m$ could be large in practice. Figure 2 illustrates the basic idea of HILL. Given a network with $n=6$ nodes, it first decomposes heterogeneous information affinity $\mathrm{S}$ into the product of $\mathbf{H}$ and $\mathbf{H}^{\top}$. Meanwhile, it imposes an edge-based penalty into this decomposition such that connected nodes are close to each other in $\mathrm{H}$, and the closeness is controlled by the edge weights in $\mathrm{W}$. The goal is to make more similar nodes in the network space to be closer in $\mathbf{H}$. To accelerate the optimization, a distributed algorithm is proposed to separate the original problem into $2 n=12$ sub-problems of low complexity. The first $n=6$ sub-problems are designed to be independent of each other, and the same as the last six. So the sub-problems could be assigned to $c=3$ workers without order. In the final output, node 1 and node 3 are represented by similar vectors [0.54, 0.27] and $[0.55,0.28]$, which indicates that they are similar to each other in the original network and heterogeneous information joint space.

\subsection{Network Topological Structure Modeling}

To render the joint embedding representation $\mathrm{H}$ well-posed, HILL learns and preserves the node proximity in both the network and heterogeneous information space. We first introduce how HILL models the network proximity via $\ell_{2}$-norm to enable robust learning.

We propose to preserve the node proximity in $\mathbf{W}$ by driving nodes with more similar topological structure or connected by higher weights to have similar vector representations. It is based on two hypotheses $[14,51]$ as follows. First, a graph-based mapping is assumed to be smooth across edges, especially for the regions of high density [8]. Second, the cluster hypothesis [35] suggests that similar nodes tend to cluster together. To achieve these goals, we propose the following loss 
function to minimize the embedding differences between all pairs of connected nodes,

$$
\mathcal{J}_{\mathcal{G}}=\sum_{(i, j) \in \mathcal{E}} w_{i j}\left\|\mathbf{h}_{i}-\mathbf{h}_{j}\right\|_{2}
$$

where rows $\mathbf{h}_{i}$ and $\mathbf{h}_{j}$ are vector representations of node $i$ and node $j$, and $w_{i j}$ is the edge weight between the two. The key idea is that, to minimize the penalty $w_{i j}\left\|\mathbf{h}_{i}-\mathbf{h}_{j}\right\|_{2}$, a larger weight $w_{i j}$ is more likely to enforce the difference of $\mathbf{h}_{i}$ and $\mathbf{h}_{j}$ to be smaller. We employ $\ell_{2}$-norm as the difference metric to alleviate the negative impacts resulted from outliers and missing data. The proposed network structure modeling enjoys several nice properties as follows. First, it is in line with the fused lasso [47] and network lasso [12], which could induce sparsity in the differences of vector representations of similar nodes, and perform continuous edges selection similar to the continuous variable selection in lasso [46]. This is in compliance with the cluster hypothesis in graphs. Second, it can be generalized to model different types of networks, such as weighted and unweighted networks. Hence, it can be easily applied to many real-world applications.

\subsection{Heterogeneous Information Proximity Modeling}

We now demonstrate how HILL makes the embedding representation $\mathbf{H}$ also well preserve the node proximity w.r.t. the heterogeneous information. Although the information in A has distinct data characteristics and structures compared with the topological information in $\mathbf{W}$, the node proximity defined by $\mathrm{A}$ and $\mathrm{W}$ would have a similar structure. Thus, we perform the heterogeneous information learning by modeling the affinity matrix of A. However, as the number of nodes $n$ scales up, this affinity matrix would increase exponentially, and may not be able to be cached on a single machine, not to mention the operations on it. A distributed framework would be necessary.

To achieve the goal, motivated by the symmetric matrix factorization [25], we propose to approximate heterogeneous information affinity matrix $\mathrm{S}$ with the product of $\mathbf{H}$ and $\mathbf{H}^{\top}$. The basic idea is to enforce the dot product of vector representations $\mathbf{h}_{i}$ and $\mathbf{h}_{j}$ to approach the corresponding heterogeneous information similarity $\mathbf{s}_{i j}$. The corresponding loss function is defined as follows:

$$
\mathcal{J}_{A}=\left\|\mathbf{S}-\mathbf{H H}^{\top}\right\|_{\mathrm{F}}^{2}=\sum_{i=1}^{n} \sum_{j=1}^{n}\left(\mathbf{s}_{i j}-\mathbf{h}_{i} \mathbf{h}_{j}^{\top}\right)^{2},
$$

where heterogeneous information affinity matrix $\mathrm{S}$ could be calculated by a representative similarity measure. For simplicity, we use cosine similarity in this article. The loss function $\mathcal{J}_{A}$ allows our framework HILL to be optimized in a distributed manner, which will be introduced in Section 6.

\subsection{Joint Embedding Representation Learning}

We have implemented two loss functions $\mathcal{J}_{\mathcal{G}}$ and $\mathcal{J}_{A}$ to model the node proximity w.r.t. the topological structure and heterogeneous information, respectively. To make them complement each other toward a joint robust and informative space, we jointly model the two types of information in a unified optimization problem defined as follows:

$$
\min _{\mathbf{H}} \mathcal{J}=\left\|\mathbf{S}-\mathbf{H H}^{\top}\right\|_{\mathrm{F}}^{2}+\lambda \sum_{(i, j) \in \mathcal{E}} w_{i j}\left\|\mathbf{h}_{i}-\mathbf{h}_{j}\right\|_{2} .
$$

Scalar $\lambda$ serves as an overall parameter that defines a trade-off between the contributions of network and heterogeneous information. It is also a regularization parameter that balances the number of clusters [12,31]. An intuitive explanation is that when $\lambda$ is close to 0 , the network topology cannot affect the final result $\mathbf{H}$, so each node can be seen as an isolated cluster. When $\lambda$ is sufficiently large, the optimal solution will end up with the same representation for all nodes, which forms a single cluster. This allows us to tune the number of clusters continuously. The number 
of cluster is often not specified in network embedding, and tunability of $\lambda$ is beneficial in this scenario.

\section{THREE REAL-WORLD APPLICATIONS OF HILL}

We now illustrate the generalizability of HILL with three real-world applications, i.e., attributed network embedding, second-order proximity learning, and link directionality modeling. We also have designed a variation of HILL for the third application.

Attributed Network Embedding. As indicated by the data analysis on the real-world datasets in Section 3, node attribute information is tightly hinged with network topological structure. Node proximity w.r.t. node attributes tends to be consistent with the one w.r.t. the network, and could be potentially helpful in learning better vector representations. Thus, we leverage the proposed framework HILL to incorporate the node attribute proximity into the network embedding. We achieve this by defining the heterogeneous information $\mathrm{A}$ as the node attribute information matrix, with $\mathbf{a}_{i}$ describes the characteristic attributes accompanied with node $i$. Proximity $s_{i j}$ denotes the cosine similarity of node $i$ 's and node $j$ 's node attributes.

Second-Order Proximity Learning. While $\mathcal{J}_{\mathcal{G}}$ in HILL could well preserve the first-order proximity, i.e., nodes with higher direct tie strengths would have similar vector representations, in practice, nodes with similar neighbors also tend to be similar. For instance, two users in Facebook sharing many common student friends are more likely to be friends in the same school. Thus, we are motivated to incorporate the second-order proximity into the embedding representation $\mathbf{H}$.

A traditional solution [44] is to concatenate the embedding representations of first-order and second-order proximities. However, it might achieve suboptimal solutions because of the heterogeneity of the two types of proximities. We achieve the second-order proximity learning by defining the heterogeneous information affinity matrix $S=S^{(W)}$, where $S^{(W)}$ is the cosine similarity of $\mathbf{W}$ and collects the second-order proximity. It is straightforward to extend to other types of similarities.

Link Directionality Modeling. We have introduced how to efficiently embed undirected networks, and now focus on directed networks, with two major scenarios as follows.

Pure Directed Networks. For directed networks without node attributes, we could treat the link directionality as the heterogeneous information and jointly embed it via HILL. Specifically, to embed the properties of outgoing links, we define $\mathrm{A} \in \mathbb{R}^{n \times n}$ as the outgoing network matrix, with row $\mathbf{a}_{i}$ collects all the outgoing links of node $i$. The corresponding objective function is defined as follows:

$$
\min _{\mathbf{H}} \mathcal{J}_{\text {Out }}=\left\|\mathbf{S}^{\text {(Out) }}-\mathbf{H} \mathbf{H}^{\top}\right\|_{\mathrm{F}}^{2}+\lambda \sum_{(i, j) \in \widetilde{\mathcal{E}}} \widetilde{w}_{i j}\left\|\mathbf{h}_{i}-\mathbf{h}_{j}\right\|_{2},
$$

where $\mathrm{S}^{(\mathrm{Out})}$ is the cosine similarity of the outgoing network matrix, and $\widetilde{w}_{i j}=\widetilde{w}_{j i}=\frac{w_{i j}+w_{j i}}{2}$ contains the pure topological structure. Set $\widetilde{\mathcal{E}}$ collects all the edges with non-zero weights $\widetilde{w}_{i j}$. It is straightforward to perform the incoming network embedding in a similar way.

Directed Attributed Networks. In social media, the relationships are often directed, with a rich set of node attributes that are also available. For instance, on Twitter, the President of USA has millions of followers, but only follows a few of them. A straightforward way to embed directed attributed networks is to discard the directionality and set edge weights $w_{i j}$ and $w_{j i}$ equal to their mean value [30]. However, it could be appealing to jointly embed the link directionality and node attributes. HILL achieves this joint learning by defining the heterogeneous information $\mathrm{A}$ as the node attribute information matrix, and defining matrix $\mathbf{W}$ as the cosine similarity of the outgoing 
network matrix $\mathbf{S}^{\text {(Out) }}$. In the optimization, $\mathbf{S}^{\text {(Out) }}$ could also be calculated in a distributed way. It is straightforward to jointly embed the incoming network and node attributes in a similar way.

\section{A DISTRIBUTED OPTIMIZATION ALGORITHM FOR ACCELERATION}

In this section, we introduce the optimization of HILL. Its objective function in Equation (3) not only could jointly model network proximity and heterogeneous information affinity, but also enjoys a specially designed structure that enables it to be optimized in an efficient and distributed manner, i.e., $\mathcal{J}$ is separable for $\mathbf{h}_{i}$ and can be reformulated as a bi-convex optimization problem. Based on this, we propose an efficient algorithm to obtain $\mathrm{H}$, with three major procedures as follows.

First, we add a copy $\mathbf{Z}=\mathbf{H}$, then the objective function in Equation (3) could be rewritten as follows:

$$
\mathcal{J}=\sum_{i=1}^{n}\left(\left\|\mathbf{s}_{i}-\mathbf{h}_{i} \mathbf{Z}^{\top}\right\|_{2}^{2}+\lambda \sum_{j \in N(i)} w_{i j}\left\|\mathbf{h}_{i}-\mathbf{z}_{j}\right\|_{2}\right)=\sum_{i=1}^{n}\left(\left\|\mathbf{s}_{i}-\mathbf{z}_{i} \mathbf{H}^{\top}\right\|_{2}^{2}+\lambda \sum_{j \in N(i)} w_{i j}\left\|\mathbf{z}_{i}-\mathbf{h}_{j}\right\|_{2}\right) .
$$

Based on this, we further reformulate Equation (3) into a linearly constrained problem defined as follows:

$$
\begin{array}{cc}
\min _{\mathbf{H}} \mathcal{J}=\sum_{i=1}^{n}\left\|\mathbf{s}_{i}-\mathbf{h}_{i} \mathbf{Z}^{\top}\right\|_{2}^{2}+\lambda \sum_{(i, j) \in \mathcal{E}} w_{i j}\left\|\mathbf{h}_{i}-\mathbf{z}_{j}\right\|_{2}, \\
\text { subject to } \\
\mathbf{h}_{i}=\mathbf{z}_{i}, \text { for } i=1, \ldots, n .
\end{array}
$$

This indicates that $\mathcal{J}$ is separable for both $\mathbf{h}_{i}$ and $\mathbf{z}_{i}$. Since $\ell_{2}$-norm is convex, it is easy to verify that the function in Equation (6) is bi-convex, i.e., convex w.r.t. $\mathbf{h}_{i}$ when $\mathbf{Z}$ is fixed and convex w.r.t. $\mathbf{z}_{i}$ when $\mathbf{H}$ is fixed. However, it is infeasible to obtain closed-form solutions for these $2 n$ sub-problems because of the linear constraint $\mathbf{H}=\mathbf{Z}$.

Second, we solve the above optimization by converting it into $2 n$ simple update steps and one simple matrix update step, motivated by the distributed convex optimization technique - Alternating Direction Method of Multipliers (ADMM) [3, 12]. The augmented Lagrangian [13] of the objective function in Equation (6) is formulated as follows:

$$
\mathcal{L}=\sum_{i=1}^{n}\left\|\mathbf{s}_{i}-\mathbf{h}_{i} \mathbf{Z}^{\top}\right\|_{2}^{2}+\lambda \sum_{(i, j) \in \mathcal{E}} w_{i j}\left\|\mathbf{h}_{i}-\mathbf{z}_{j}\right\|_{2}+0.5 \rho \sum_{i=1}^{n}\left(\left\|\mathbf{h}_{i}-\mathbf{z}_{i}+\mathbf{u}_{i}\right\|_{2}^{2}-\left\|\mathbf{u}_{i}\right\|_{2}^{2}\right),
$$

where rows $\mathbf{u}_{1}, \ldots, \mathbf{u}_{n} \in \mathbb{R}^{d}$ are the scaled dual variables [3], and $\rho>0$ is the penalty parameter. The minimizer of Equation (6) is then converted to the saddle point of $\mathcal{L}$, which can be approached by iteratively finding the optimal $\mathrm{H}, \mathrm{Z}$, and $\mathrm{U}$.

Third, assume that, in iteration $t$, we have obtained the optimum of the three matrices as $\mathbf{H}^{t}$, $\mathbf{Z}^{t}$, and $\mathbf{U}^{t}$. Then, in iteration $t+1$, the calculation of $\mathbf{H}^{t+1}$ or $\mathbf{Z}^{t+1}$ could be separated into $n$ independent and simple sub-problems as follows:

$$
\left\{\begin{array}{l}
\mathbf{h}_{i}^{t+1}=\underset{\mathbf{h}_{i}}{\operatorname{argmin}}\left(\left\|\mathbf{s}_{i}-\mathbf{h}_{i} \mathbf{Z}^{t^{\top}}\right\|_{2}^{2}+\lambda \sum_{j \in N(i)} w_{i j}\left\|\mathbf{h}_{i}-\mathbf{z}_{j}^{t}\right\|_{2}+0.5 \rho\left\|\mathbf{h}_{i}-\mathbf{z}_{i}^{t}+\mathbf{u}_{i}^{t}\right\|_{2}^{2}\right), \\
\mathbf{z}_{i}^{t+1}=\underset{\mathbf{z}_{i}}{\operatorname{argmin}}\left(\left\|\mathbf{s}_{i}-\mathbf{z}_{i} \mathbf{H}^{t+1^{\top}}\right\|_{2}^{2}+\lambda \sum_{j \in N(i)} w_{j i}\left\|\mathbf{z}_{i}-\mathbf{h}_{j}^{t+1}\right\|_{2}+0.5 \rho\left\|\mathbf{z}_{i}-\mathbf{h}_{i}^{t+1}-\mathbf{u}_{i}^{t}\right\|_{2}^{2}\right),
\end{array}\right.
$$

The update rule for $\mathbf{U}^{t+1}$ is defined as follows:

$$
\mathrm{U}^{t+1}=\mathbf{U}^{t}+\left(\mathbf{H}^{t+1}-\mathbf{Z}^{t+1}\right) .
$$


We need to obtain all the $\mathbf{h}_{i}^{t+1}$ before calculating $\mathbf{z}_{i}^{t+1}$, and update $\mathbf{U}^{t+1}$ after getting all the $\mathbf{h}_{i}^{t+1}$ and $\mathbf{z}_{i}^{t+1}$. However, the order of solving $\mathbf{h}_{i}^{t+1}$, for $i=1, \ldots, n$, is not fixed, since they are independent of each other. When the machine capacity is limited, $s_{i}$ could be calculated separately in each corresponding worker via equation $\mathbf{s}_{i}=\mathbf{a}_{i} \mathbf{A}^{\top} \oslash\left(q_{i} \mathbf{q}\right)$, where notation $\oslash$ denotes the elementwise division. Vector $\mathrm{q}$ is the dot product of each heterogeneous information vector and itself, i.e., $q=\left[\sqrt{\mathbf{a}_{1} \mathbf{a}_{1}^{\top}}, \ldots, \sqrt{\mathbf{a}_{n} \mathbf{a}_{n}^{\top}}\right]$. Next, we introduce how to solve the problems in Equations (8) and (9).

\subsection{Computation of $\mathrm{h}_{i}^{t+1}$ and $\mathrm{z}_{i}^{t+1}$}

Although the function in Equation (8) is convex, it is challenging to get the closed-form solution. We propose to approach the optimal $\mathbf{h}_{i}^{t+1}$ iteratively. There are several non-differentiable points at $\mathbf{h}_{i}=\mathbf{z}_{j}^{t}$ in Equation (8), and the classical solution is to use subgradient methods. However, these methods usually converge slowly. Thus, we present an efficient heuristic approach to calculating $\mathbf{h}_{i}^{t+1}$.

We first define $\mathbf{h}_{i}^{k}=\mathbf{h}_{i}^{t}$ as the initial point, with $k=0$. Then, by taking the derivative of the function in Equation (8) w.r.t. $\mathbf{h}_{i}$, and setting it to zero, we get an update rule for $\mathbf{h}_{i}^{k+1}$ as follows:

$$
\mathbf{h}_{i}^{k+1}=\left(2 \mathbf{s}_{i} \mathbf{Z}^{t}+\lambda \sum_{j \in \widetilde{N}(i)} \frac{w_{i j} \mathbf{z}_{j}^{t}}{\left\|\mathbf{h}_{i}^{k}-\mathbf{z}_{j}^{t}\right\|_{2}}+\rho \mathbf{z}_{i}^{t}-\rho \mathbf{u}_{i}^{t}\right) \times\left[2 \mathbf{Z}^{t^{\top}} \mathbf{Z}^{t}+\left(\lambda \sum_{j \in \widetilde{N}(i)} \frac{w_{i j}}{\left\|\mathbf{h}_{i}^{k}-\mathbf{z}_{j}^{t}\right\|_{2}}+\rho\right) \mathbf{I}\right]^{\dagger}
$$

where $\widetilde{N}(i)$ is the set of adjacent nodes of node $i$ with representations in $\mathbf{Z}^{t}$ not equal to $\mathbf{h}_{i}^{k}$, i.e.,

$$
\widetilde{N}(i)=\left\{j \in N(i) \text { and } z_{j}^{t} \neq \mathbf{h}_{i}^{k}\right\} .
$$

Here, $\widetilde{N}(i)$ is defined to handle non-differentiable points. We employ $\left\|\mathbf{h}_{i}^{k}-\mathbf{z}_{j}^{t}\right\|_{2}$ to estimate the distance $\left\|\mathbf{h}_{i}^{k+1}-\mathbf{z}_{j}^{t}\right\|_{2}$, and the monotonically decreasing property of the update rule in Equation (11) is proved in Theorem 1 and Corollary 1. It is proved that the objective function in Equation (8) is optimal if and only if $\mathbf{h}_{i}^{k}=\mathbf{h}_{i}^{k+1}$ or $\mathbf{h}_{i}=\mathbf{z}_{j}^{t}$. Thus, we stop updating when they are close enough, and set the final one as $\mathbf{h}_{i}^{t+1}$. The proposed solution is heuristic because in a few extreme cases, $\mathbf{h}_{i}^{t}$ might equal to $\mathbf{z}_{j}^{t}$. We could get $\mathbf{z}_{i}^{t+1}$ in a similar way, with $\hat{N}(i)=\left\{j \in N(i)\right.$ and $\left.h_{j}^{t+1} \neq \mathbf{z}_{i}^{k}\right\}$, and the corresponding update rule of $\mathbf{z}_{i}^{k+1}$ is defined as follows:

$$
\mathbf{z}_{i}^{k+1}=\left(2 \mathbf{s}_{i} \mathbf{H}^{t+1}+\lambda \sum_{j \in \hat{N}(i)} \frac{w_{i j} \mathbf{h}_{j}^{t+1}}{\left\|\mathbf{z}_{i}^{k}-\mathbf{h}_{j}^{t+1}\right\|_{2}}+\rho \mathbf{h}_{i}^{t+1}+\rho \mathbf{u}_{i}^{t}\right) \times\left[2 \mathbf{H}^{t+1}{ }^{\top} \mathbf{H}^{t+1}+\left(\lambda \sum_{j \in \hat{N}(i)} \frac{w_{i j}}{\left\|\mathbf{z}_{i}^{k}-\mathbf{h}_{j}^{t+1}\right\|_{2}}+\rho\right) \mathbf{I}\right]^{\dagger} .
$$

Theorem 1 (Monotonicity of Update Rules). For any finite number of known vectors $\mathbf{z}_{j} \in$ $\mathbb{R}^{1 \times d}$ and $a_{j}$, and known $\mathrm{Q} \in \mathbb{R}^{d \times d}$ and $\mathbf{c} \in \mathbb{R}^{1 \times d}$, given a function of $\mathbf{x} \in \mathbb{R}^{1 \times d}$ defined as follows:

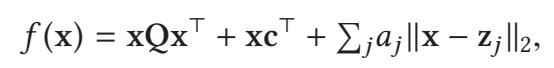

we have $f\left(\mathbf{x}^{k+1}\right) \leq f\left(\mathbf{x}^{k}\right)$ for any pair of $\left\{\mathbf{x}^{k}, \mathbf{x}^{k+1}\right\}$ satisfying the rule as follows, with $\mathbf{x}^{k} \neq \mathbf{z}_{j}$,

$$
\mathbf{x}^{k+1}=\left(\sum_{j} a_{j} b_{j} \mathbf{z}_{j}-\mathbf{c}\right) \times\left(2 \mathbf{Q}+\sum_{j} a_{j} b_{j} \mathbf{I}\right)^{\dagger},
$$

where $b_{j}=\frac{1}{\left\|\mathbf{x}^{k}-\mathbf{z}_{j}\right\|_{2}}$. Furthermore, $f(\mathbf{x})$ is optimum if and only if $\mathbf{x}^{k+1}=\mathbf{x}^{k}$ or $\mathbf{x}=\mathbf{z}_{j}$. 
Proof. Our goal is to prove that $f\left(\mathbf{x}^{k+1}\right) \leq f\left(\mathbf{x}^{k}\right)$, when $\mathbf{x}^{k+1}$ and $\mathbf{x}^{k}$ have a relation as follows:

$$
\mathbf{x}^{k+1}=\left(\sum_{j} a_{j} b_{j} \mathbf{Z}_{j}-\mathbf{c}\right) \times\left(2 \mathbf{Q}+\sum_{j} a_{j} b_{j} \mathbf{I}\right)^{\dagger} .
$$

First, for $\mathbf{x}^{k} \neq \mathbf{z}_{j}$, we define a new quadratic function of $\mathbf{x}$ as follows:

$$
g(\mathbf{x}) \triangleq \mathbf{x}\left(\mathbf{Q}+0.5 \sum_{j} a_{j} b_{j} \mathbf{I}\right) \mathbf{x}^{\top}-\left(\sum_{j} a_{j} b_{j} \mathbf{z}_{j}-\mathbf{c}\right) \mathbf{x}^{\top} .
$$

It is easy to prove that $g(\mathbf{x})$ achieves the global minimum at $\mathbf{x}^{k+1}$. Thus, for any vector $\mathbf{x}^{k} \neq \mathbf{z}_{j}$, we have $g\left(\mathbf{x}^{k+1}\right) \leq g\left(\mathbf{x}^{k}\right)$. By substituting $\mathbf{x}^{k+1}$ and $\mathbf{x}^{k}$ into $g(\mathbf{x})$, we get an inequation as follows:

$$
\mathbf{x}^{k+1} \mathbf{Q} \mathbf{x}^{k+1^{\top}}+\mathbf{c} \mathbf{x}^{k+1^{\top}}+\sum_{j} a_{j} b_{j}\left(0.5 \mathbf{x}^{k+1}-\mathbf{z}_{j}\right) \mathbf{x}^{k+1^{\top}} \leq \mathbf{x}^{k} \mathbf{Q} \mathbf{x}^{k^{\top}}+\mathbf{c} \mathbf{x}^{k^{\top}}+\sum_{j} a_{j} b_{j}\left(0.5 \mathbf{x}^{k}-\mathbf{z}_{j}\right) \mathbf{x}^{k^{\top}}
$$

Second, for any pair of vectors $\left\{\mathbf{x}^{k}, \mathbf{x}^{k+1}\right\}$, w.r.t. any $\mathbf{z}_{j}$, we have,

$$
0.5\left\|\mathbf{x}^{k}-\mathbf{z}_{j}\right\|_{2}^{2}+0.5\left\|\mathbf{x}^{k+1}-\mathbf{z}_{j}\right\|_{2}^{2} \geq\left\|\mathbf{x}^{k}-\mathbf{z}_{j}\right\|_{2}\left\|\mathbf{x}^{k+1}-\mathbf{z}_{j}\right\|_{2} .
$$

We could further unfold Equation (16) as follows:

$$
\begin{aligned}
\left(0.5 \mathbf{x}^{k} \mathbf{x}^{k^{\top}}-\mathbf{z}_{j} \mathbf{x}^{k^{\top}}+0.5 \mathbf{z}_{j} \mathbf{z}_{j}^{\top}\right)+0.5 \mathbf{z}_{j} \mathbf{z}_{j}^{\top} & \geq\left\|\mathbf{x}^{k}-\mathbf{z}_{j}\right\|_{2}\left\|\mathbf{x}^{k+1}-\mathbf{z}_{j}\right\|_{2}-0.5 \mathbf{x}^{k+1} \mathbf{x}^{k+1}{ }^{\top}+\mathbf{z}_{j} \mathbf{x}^{k+1^{\top}}, \\
\mathbf{x}^{k} \mathbf{x}^{k^{\top}}-2 \mathbf{z}_{j} \mathbf{x}^{k^{\top}}+\mathbf{z}_{j} \mathbf{z}_{j}^{\top}-0.5 \mathbf{x}^{k} \mathbf{x}^{k^{\top}}+\mathbf{z}_{j} \mathbf{x}^{k^{\top}} & \geq\left\|\mathbf{x}^{k}-\mathbf{z}_{j}\right\|_{2}\left\|\mathbf{x}^{k+1}-\mathbf{z}_{j}\right\|_{2}-0.5 \mathbf{x}^{k+1} \mathbf{x}^{k+1}+\mathbf{z}_{j} \mathbf{x}^{k+1}{ }^{\top}, \\
\left\|\mathbf{x}^{k}-\mathbf{z}_{j}\right\|_{2}^{2}-0.5 \mathbf{x}^{k} \mathbf{x}^{k^{\top}}+\mathbf{z}_{j} \mathbf{x}^{k^{\top}} & \geq\left\|\mathbf{x}^{k}-\mathbf{z}_{j}\right\|_{2}\left\|\mathbf{x}^{k+1}-\mathbf{z}_{j}\right\|_{2}-0.5 \mathbf{x}^{k+1} \mathbf{x}^{k+1}+\mathbf{z}_{j} \mathbf{x}^{k+1}{ }^{\top}, \\
\left\|\mathbf{x}^{k}-\mathbf{z}_{j}\right\|_{2}\left\|\mathbf{x}^{k+1}-\mathbf{z}_{j}\right\|_{2}-\left(0.5 \mathbf{x}^{k+1}-\mathbf{z}_{j}\right) \mathbf{x}^{k+1} & \leq\left\|\mathbf{x}^{k}-\mathbf{z}_{j}\right\|_{2}^{2}-\left(0.5 \mathbf{x}^{k}-\mathbf{z}_{j}\right) \mathbf{x}^{k^{\top}} .
\end{aligned}
$$

Since $b_{j}=\frac{1}{\left\|\mathbf{x}^{k}-\mathbf{z}_{j}\right\|_{2}}$, we could multiply $b_{j}$ on both sides of the above inequation, and have

$$
\begin{gathered}
\left\|\mathbf{x}^{k+1}-\mathbf{z}_{j}\right\|_{2}-b_{j}\left(0.5 \mathbf{x}^{k+1}-\mathbf{z}_{j}\right) \mathbf{x}^{k+1^{\top}} \leq\left\|\mathbf{x}^{k}-\mathbf{z}_{j}\right\|_{2}-b_{j}\left(0.5 \mathbf{x}^{k}-\mathbf{z}_{j}\right) \mathbf{x}^{k^{\top}}, \\
\sum_{j} a_{j}\left\|\mathbf{x}^{k+1}-\mathbf{z}_{j}\right\|_{2}-\sum_{j} a_{j} b_{j}\left(0.5 \mathbf{x}^{k+1}-\mathbf{z}_{j}\right) \mathbf{x}^{k+1^{\top}} \\
\leq \sum_{j} a_{j}\left\|\mathbf{x}^{k}-\mathbf{z}_{j}\right\|_{2}-\sum_{j} a_{j} b_{j}\left(0.5 \mathbf{x}^{k}-\mathbf{z}_{j}\right) \mathbf{x}^{k^{\top}} .
\end{gathered}
$$

By summing up Equations (15) and (17), we get

$$
f\left(\mathbf{x}^{k+1}\right) \leq f\left(\mathbf{x}^{k}\right) .
$$

Based on this proved monotonicity, $f(\mathbf{x})$ is guaranteed to achieve the global optimum if and only if $\mathbf{x}^{k+1}=\mathbf{x}^{k}$ or $\mathbf{x}=\mathbf{z}_{j}$, since it is convex.

COROLlary 1. The update rule in Equation (11) will monotonically decrease the objective function in Equation (8), and converge to the global optimum if and only if $\mathbf{h}_{i}^{k+1}=\mathbf{h}_{i}^{k}$ or $\mathbf{h}_{i}=\mathbf{z}_{j}^{t}$.

Proof. Based on Theorem 1, it is straightforward to prove Corollary 1 by defining the corresponding known $\mathrm{Q}$, c, and $a_{j}$, for all the $j \in N(i)$, as follows:

$$
\begin{gathered}
\mathrm{Q}=\mathrm{Z}^{t^{\top}} \mathbf{Z}^{t}+0.5 \rho \mathbf{I}, \\
\mathbf{c}=\rho \mathbf{u}_{i}^{t}-\rho \mathbf{z}_{i}^{t}-2 \mathbf{s}_{i} \mathbf{Z}^{t}, \\
a_{j}=\lambda w_{i j} .
\end{gathered}
$$




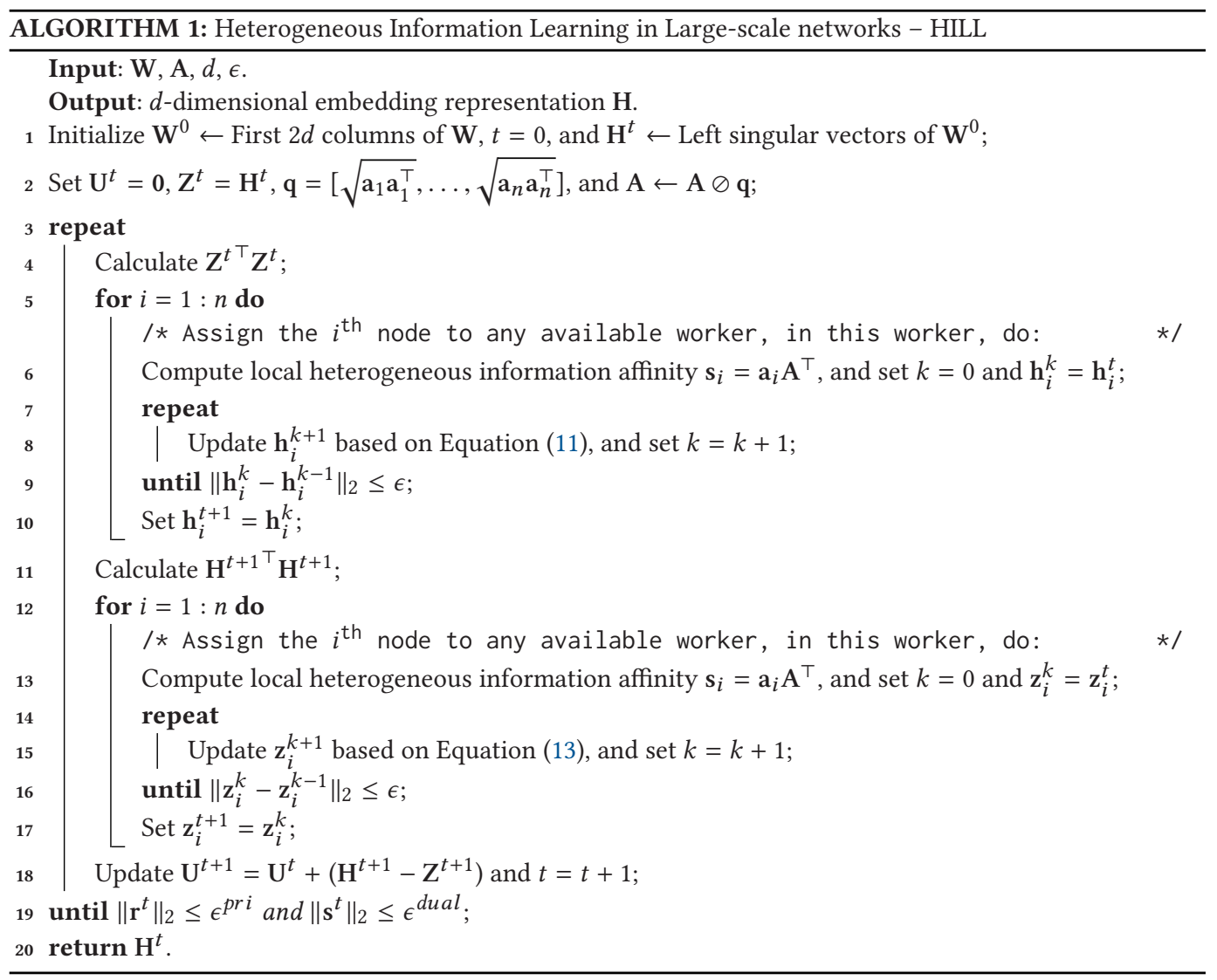

\subsection{Summary of the Optimization of HILL}

The distributed algorithm for optimizing the problem in our framework HILL is described in Algorithm 1. To have an appropriate initialization, we set the initial embedding representation $\mathbf{H}^{t=0}$ as the left singular vectors of $\mathbf{W}^{0}$, where $\mathbf{W}^{0}$ is a matrix that randomly samples $2 d$ columns of $\mathbf{W}$. We update $\mathbf{H}$ and $\mathbf{Z}$ iteratively until they are approximately equivalent and no longer change much in one iteration. The corresponding termination criterion is that primal residual $\mathbf{r}^{t}=\sum_{i=1}^{n}\left(\mathbf{h}_{i}^{t}-\mathbf{z}_{i}^{t}\right)$ and dual residual $\mathbf{s}^{t}=-\rho \sum_{i=1}^{n}\left(\mathbf{z}_{i}^{t}-\mathbf{z}_{i}^{t-1}\right)$ should be sufficiently small [3]. To find $\mathbf{H}^{t+1}$, the optimization process is split into $n$ number of sub-problems. These $n$ update steps of $\mathbf{h}_{i}^{t+1}$ could be assigned to $c$ workers in a distributed way as illustrated in Figure 2. We initialize $\mathbf{h}_{i}^{k}=\mathbf{h}_{i}^{t}$ and keep applying the update rule on $\mathbf{h}_{i}^{k+1}$ until it convergences, and the final result is set to be the new $\mathbf{h}_{i}^{t+1}$. Variable matrix $\mathbf{Z}^{t+1}$ is calculated in a similar way. $\mathbf{H}$ and $\mathbf{Z}$ are non-separable in Equation (6), and the convergence of non-separable biconvex ADMM is still an open problem, with few attempts recently [10].

The proposed framework HILL enjoys several nice properties as follows. First, it enables the $n$ update steps for $\mathbf{h}_{i}^{t+1}$ (or $\mathbf{z}_{i}^{t+1}$ ), for $i=1, \ldots, n$, independent of each other. Thus, in each iteration, the global coordination could assign these tasks to available workers and collect the solutions from them without a fixed order. Second, all these small update steps have low complexity and converge fast. In such way, we split the original complex embedding problem into $2 n$ simple convex optimization sub-problems. Third, as it is typical for ADMM [3], Algorithm 1 tends to converge to 
a modest accuracy in a few iterations. The update of $\mathbf{h}_{i}^{k+1}$ (or $\mathbf{z}_{i}^{k+1}$ ) also converges rapidly, since the difference between $\left\|\mathbf{h}_{i}^{k}-\mathbf{z}_{j}^{t}\right\|_{2}$ and $\left\|\mathbf{h}_{i}^{k+1}-\mathbf{z}_{j}^{t}\right\|_{2}$ is always small.

Complexity Analysis. In the initialization, the time complexity for calculating singular vectors of an $n$ by $d$ matrix $\mathbf{W}^{0}$ is $O\left(d^{2} n\right)$. We denote the number of operations required to obtain the heterogeneous information affinity matrix $\mathrm{S}$ as $\mathcal{T}_{\mathrm{S}}$. In each sub-problem, since we only need to compute $\mathrm{Z}^{t^{\top}} \mathbf{Z}^{t}$ once for all $\mathbf{h}_{i}^{t+1}$ per iteration, the update time for $\mathbf{h}_{i}$ should be $O\left(d^{3}+d n+d|N(i)|\right)$. Since $d \ll n$, this complexity could be reduced to $O(n)$. Therefore, the total time complexity of HILL is $O\left(n+\mathcal{T}_{\mathrm{S}}+\frac{n^{2}}{c}\right)$, which equals to $O\left(n \mathcal{N}_{\mathrm{A}}+\frac{n^{2}}{c}\right)$, where $\mathcal{N}_{\mathrm{A}}$ is the number of non-zero entries in A. It should be noted that we avoid the computation of the similarity matrix of $\mathbf{W}$, which saves lots of time. Except for the space for storing the original $\mathbf{W}$ and $\mathbf{A}$ in the coordinator, it is easy to check that the space complexity of HILL is only $O(n)$. Because the rule in Equation (11) shows that, when calculating $\mathbf{H}$, only the low-dimensional matrix $\mathbf{Z}$ is replicated in each worker.

\subsection{Heterogeneous Information Learning in Streaming Networks}

The proposed framework HILL could also handle streaming networks. Given an optimal joint embedding representation $\mathbf{H}^{*}$, we could easily acquire the vector representations of newly included nodes. Assume we have a new node $i$ with network features $\mathbf{w}_{i}$ and heterogeneous information $\mathbf{a}_{i}$, we could obtain $\mathbf{h}_{i}$ readily via the objective function as follows:

$$
\mathbf{h}_{i}=\underset{\mathbf{h}_{i}}{\operatorname{argmin}}\left\|\mathbf{s}_{i}-\mathbf{h}_{i} \hat{\mathbf{H}}^{\top}\right\|_{2}^{2}+2 \lambda \sum_{j \in N(i)} w_{i j}\left\|\mathbf{h}_{i}-\mathbf{h}_{j}^{*}\right\|_{2},
$$

where $\hat{\mathbf{H}}$ is a matrix that concatenates $\mathbf{H}^{*}$ and $\mathbf{h}_{i}$. Similarly, we could make a copy $\mathbf{z}_{i}=\mathbf{h}_{i}$ and approach the optimal solution of Equation (22) via three sub-problems as follows:

$$
\left\{\begin{array}{l}
\mathbf{h}_{i}^{t+1}=\underset{\mathbf{h}_{i}}{\operatorname{argmin}}\left(\left\|\mathbf{s}_{i}-\mathbf{h}_{i} \hat{\mathbf{Z}}^{t^{\top}}\right\|_{2}^{2}+\lambda \sum_{j \in N(i)} w_{i j}\left\|\mathbf{h}_{i}-\mathbf{h}_{j}^{*}\right\|_{2}+0.5 \rho\left\|\mathbf{h}_{i}-\mathbf{z}_{i}^{t}+\mathbf{u}_{i}^{t}\right\|_{2}^{2}\right), \\
\mathbf{z}_{i}^{t+1}=\underset{\mathbf{z}_{i}}{\operatorname{argmin}}\left(\left\|1-\mathbf{z}_{i} \mathbf{h}_{i}^{t+1^{\top}}\right\|_{2}^{2}+\lambda \sum_{j \in N(i)} w_{j i}\left\|\mathbf{z}_{i}-\mathbf{h}_{j}^{*}\right\|_{2}+0.5 \rho\left\|\mathbf{z}_{i}-\mathbf{h}_{i}^{t+1}-\mathbf{u}_{i}^{t}\right\|_{2}^{2}\right), \\
\mathbf{u}_{i}^{t+1}=\mathbf{u}_{i}^{t}+\left(\mathbf{h}_{i}^{t+1}-\mathbf{z}_{i}^{t+1}\right),
\end{array}\right.
$$

where $\hat{\mathbf{Z}}^{t}$ is a matrix that concatenates $\mathbf{H}^{*}$ and $\mathbf{z}_{i}^{t}$. Based on Theorem 1, it is straightforward to find the following update rules to iteratively solve the sub-problems in Equation (23).

$$
\left\{\begin{array}{l}
\mathbf{h}_{i}^{k+1}=\left(2 \mathbf{s}_{i} \hat{\mathbf{Z}}^{t}+\lambda \sum_{j \in N(i)} \frac{w_{i j} \mathbf{h}_{j}^{*}}{\left\|\mathbf{h}_{i}^{k}-\mathbf{h}_{j}^{*}\right\|_{2}}+\rho \mathbf{z}_{i}^{t}-\rho \mathbf{u}_{i}^{t}\right) \times\left[2 \hat{\mathbf{Z}}^{\top} \hat{\mathbf{Z}}^{t}+\left(\lambda \sum_{j \in N(i)} \frac{w_{i j}}{\left\|\mathbf{h}_{i}^{k}-\mathbf{h}_{j}^{*}\right\|_{2}}+\rho\right) \mathbf{I}\right]^{\dagger}, \\
\mathbf{z}_{i}^{k+1}=\left(2 \mathbf{h}_{i}^{t+1}+\lambda \sum_{j \in N(i)} \frac{w_{i j} \mathbf{h}_{j}^{*}}{\left\|\mathbf{z}_{i}^{k}-\mathbf{h}_{j}^{*}\right\|_{2}}+\rho \mathbf{h}_{i}^{t+1}+\rho \mathbf{u}_{i}^{t}\right) \times\left[2 \mathbf{h}_{i}^{t+1} \mathbf{h}_{i}^{t+1}+\left(\lambda \sum_{j \in N(i)} \frac{w_{i j}}{\left\|\mathbf{z}_{i}^{k}-\mathbf{h}_{j}^{*}\right\|_{2}}+\rho\right) \mathbf{I}\right]^{\dagger} .
\end{array}\right.
$$

\section{EXPERIMENTS}

In this section, we empirically evaluate the effectiveness and efficiency of the proposed framework HILL. We aim at answering three research questions as follows. (1) What is the impact of the heterogeneous information such as node attributes and second-order proximity on the network embedding? (2) How effective and efficient is HILL compared with the state-of-the-art heterogeneous information joint learning methods? (3) What are the impacts of the three parameters $\lambda, \rho$, and $d$ on the performance of HILL? 


\subsection{Baseline Methods}

We apply the proposed framework HILL to two tasks, i.e., attributed network embedding and second-order proximity learning, and get HILL_Attri and HILL_Net, respectively. It should be noted that HILL_Net only uses network information. HILL_Attri and HILL_Net are compared with three categories of baseline methods. First, to evaluate the impact of incorporating the heterogeneous information, two state-of-the-art scalable network embedding methods, i.e., DeepWalk and LINE, are used for comparison. Second, to study the contribution of incorporating the network structure, we include two methods for modeling pure node attributes, i.e., PCA and Spectral. Third, to investigate the effectiveness and efficiency of HILL_Attri, we compare it with two state-of-theart heterogeneous information joint learning methods, i.e., LCMF and MultiSpec. The detailed descriptions of these methods are listed as follows:

-DeepWalk [40]: It performs truncated random walks on a network, and embeds the walking tracks as sentences via language modeling techniques. Each vertex corresponds to a word.

- LINE [44]: It samples and embeds both one-hop and two-hop neighbors of each node, and then concatenates the two learned low-dimensional representations into one.

-HILL_Net: It treats the second-order proximity as the heterogeneous information, and employs HILL to incorporate it into the first-order proximity. Node attributes are not used in it.

$-P C A$ [22]: It is a classical dimensionality reduction technique. It takes the top $d$ principal components of the node attribute information matrix as the learned representation.

- Spectral [51]: It embeds node attributes via two steps. First, it constructs a new graph with the cosine similarity of two nodes' attribute vectors $\mathbf{a}_{i}$ and $\mathbf{a}_{j}$ as the corresponding edge weight. Second, it performs normalized spectral embedding on the constructed graph to learn $\mathrm{H}$.

- LCMF [58]: It learns a low-dimensional representation from the heterogeneous linkage and content information by carrying out a joint matrix factorization.

-MultiSpec [26]: It treats the network structure and node attributes as two views, and embeds them jointly by co-regularizing spectral clustering hypotheses across the two views.

- HILL_Attri: It treats node attributes as the heterogeneous information, and employs HILL to incorporate it into the network embedding.

- HILL_Stream: It has similar settings to HILL_Attri, except that nodes in the test group arrive one by one. It employs the objective function in Equation (22) to calculate the joint embedding representations of the streaming nodes.

\subsection{Experimental Setup}

Following the widely adopted way of validating network embedding [40, 44], we evaluate HILL_Attri, HILL_Net, and baseline methods on the node classification task [42, 58]. The goal is to predict which category or categories a new node belongs to based on its low-dimensional representation and the learned classifier. We now introduce the experimental settings in detail.

We employ five-fold cross-validation, i.e., randomly separate the entire nodes into a training group ( $\left.\mathbf{W}_{\text {train }}, \mathbf{A}_{\text {train }}, \mathbf{Y}_{\text {train }}\right)$ and a test group $\left(\mathbf{W}_{\text {test }}, \mathbf{A}_{\text {test }}, \mathbf{Y}_{\text {test }}\right)$, where $\mathbf{Y}$ denotes the labels. The edges between training group and test group are kept. To investigate the performance of a method, we apply it to both groups and learn vector representations $\mathbf{H}$ for all nodes, including $\mathbf{H}_{\text {train }}$ and $\mathbf{H}_{\text {test }}$. Since there are multiple label categories, we build a binary SVM classifier for each category based on $\mathbf{H}_{\text {train }}$ and $\mathrm{Y}_{\text {train }}$. At last, we perform the classification based on $\mathbf{H}_{\text {test }}$ and the learned SVM classifiers. The labels in $\mathrm{Y}_{\text {test }}$ serve as the ground truth. 
The classification performance is measured via two commonly used evaluation criteria, microaverage and macro-average [21]. F-measure is a widely used metric for binary classification. Microaverage is the harmonic mean of average precision and average recall, i.e.,

$$
\text { Micro-average }=\frac{\sum_{i=1}^{\ell} 2 \mathrm{TP}^{(i)}}{\sum_{i=1}^{\ell}\left(2 \mathrm{TP}^{(i)}+\mathrm{FP}^{(i)}+\mathrm{FN}^{(i)}\right)},
$$

where $\mathrm{TP}^{(i)}, \mathrm{FP}^{(i)}$, and $\mathrm{FN}^{(i)}$ denote the numbers of true positives, false positives, and false negatives in the $i$ th label category correspondingly. Macro-average is defined as an arithmetic average of $\mathrm{F}$-measure of all $\ell$ label categories, i.e.,

$$
\text { Macro-average }=\frac{1}{\ell} \sum_{i=1}^{\ell} \frac{2 \mathrm{TP}^{(i)}}{2 \mathrm{TP}^{(i)}+\mathrm{FP}^{(i)}+\mathrm{FN}^{(i)}} .
$$

We follow the suggestions of the original papers to set the parameters of baseline methods. If it is not specified, the embedding dimension $d$ is set to be 100. All experimental results are the arithmetic average of 10 test runs. We ran the experiments on a Dell OptiPlex 9030 i7-16GB desktop.

\subsection{Impact of the Heterogeneous Information}

To answer the first question proposed at the beginning of this section, i.e., how node attributes could advance the embedding representation, we compare HILL_Attri with the three network embedding methods, i.e., DeepWalk, LINE, and HILL_Net, and the two node attribute modeling methods, i.e., PCA and Spectral. To study the impact of the second-order proximity, we compare HILL_Net with DeepWalk and LINE. Since PCA and Spectral are not practicable on the Yelp dataset, we randomly select $20 \%$ of Yelp and set it as a new dataset, i.e., Yelp-sub, such that we are able to know the performance of all methods on it. The classification performance w.r.t. these methods is presented in Table 4. The experimental results of HILL_Attri and the two scalable embedding methods on the original Yelp dataset are shown in Table 5.

Form the results in Tables 4 and 5, we have three major observations as follows. First, by taking advantage of node attributes, HILL_Attri achieves significantly better performance than all network embedding methods on all datasets. For example, on BlogCatalog, we observe that incorporating node attributes allows HILL_Attri to achieve 38.7\% gain over DeepWalk and $36.3 \%$ gain over LINE in terms of Micro-average score. Second, by performing joint embedding on the network and node attributes, HILL_Attri consistently outperforms PCA and Spectral on all datasets. For example, on BlogCatalog, HILL_Attri achieves 8.8\% of improvements than PCA. Third, by incorporating the second-order proximity, HILL_Net outperforms DeepWalk. For instance, on Flickr, HILL_Net achieves a gain of $17.0 \%$ over DeepWalk. HILL_Net does not perform well on Yelp-sub since its network information is too noisy.

We also vary the number of nodes that is used for training as $\{10 \%, 25 \%, 50 \%, 100 \%\}$ of the entire training group to evaluate the effect brought by different sizes of training data. The results are shown in Table 4. From the results, similar observations are made. HILL_Attri consistently achieves better performance than all baselines on all datasets. For example, on Flickr, when training set percentage is 50\%, HILL_Attri achieves 5.0\% of improvements than Spectral.

\subsection{Effectiveness Evaluation}

To answer the second question asked at the beginning of this section, two types of comparisons are conducted. First, to study the performance of our framework on the node attribute learning, we compare HILL_Attri with the two heterogeneous information joint learning methods, i.e., LCMF 
Table 4. The Classification Performance of Different Methods on Different Datasets with $d=100$

\begin{tabular}{|c|c|c|c|c|c|c|c|c|c|c|c|c|c|}
\hline \multirow{2}{*}{\multicolumn{2}{|c|}{ Training Set Percentage }} & \multicolumn{4}{|c|}{ BlogCatalog } & \multicolumn{4}{|c|}{ Flickr } & \multicolumn{4}{|c|}{ Yelp-sub } \\
\hline & & $10 \%$ & $25 \%$ & $50 \%$ & $100 \%$ & $10 \%$ & $25 \%$ & $50 \%$ & $100 \%$ & $10 \%$ & $25 \%$ & $50 \%$ & $100 \%$ \\
\hline \# nodes & s for embedding & 1,455 & 2,079 & 3,118 & 5,196 & 2,118 & 3,026 & 4,538 & 7,564 & 13,945 & 19,921 & 29,881 & 49,802 \\
\hline \multirow{9}{*}{$\begin{array}{l}\text { Micro- } \\
\text { average }\end{array}$} & DeepWalk & 0.491 & 0.551 & 0.611 & 0.672 & 0.312 & 0.373 & 0.465 & 0.535 & 0.302 & 0.310 & 0.318 & 0.350 \\
\hline & LINE & 0.433 & 0.545 & 0.624 & 0.684 & 0.259 & 0.332 & 0.421 & 0.516 & 0.230 & 0.243 & 0.264 & 0.294 \\
\hline & HILL_Net & 0.556 & 0.628 & 0.690 & 0.747 & 0.315 & 0.397 & 0.496 & 0.626 & 0.369 & 0.373 & 0.274 & 0.247 \\
\hline & PCA & 0.695 & 0.782 & 0.823 & 0.857 & 0.508 & 0.606 & 0.666 & 0.692 & 0.667 & 0.674 & 0.681 & N.A. \\
\hline & Spectral & 0.717 & 0.791 & 0.841 & 0.869 & 0.698 & 0.771 & 0.813 & 0.846 & 0.670 & 0.683 & N.A. & N.A. \\
\hline & LCMF & 0.778 & 0.849 & 0.888 & 0.902 & 0.576 & 0.676 & 0.725 & 0.749 & 0.668 & 0.680 & 0.686 & N.A. \\
\hline & MultiSpec & 0.678 & 0.788 & 0.849 & 0.896 & 0.589 & 0.720 & 0.800 & 0.859 & 0.654 & 0.667 & N.A. & N.A. \\
\hline & HILL_Attri & 0.841 & 0.878 & 0.913 & 0.932 & 0.740 & 0.811 & 0.854 & 0.885 & 0.679 & 0.694 & 0.703 & 0.711 \\
\hline & HILL_Stream & 0.770 & 0.822 & 0.887 & 0.914 & 0.568 & 0.726 & 0.816 & 0.859 & 0.554 & 0.577 & 0.665 & 0.691 \\
\hline \multirow{9}{*}{$\begin{array}{l}\text { Macro- } \\
\text { average }\end{array}$} & DeepWalk & 0.489 & 0.548 & 0.606 & 0.665 & 0.310 & 0.371 & 0.462 & 0.530 & 0.139 & 0.159 & 0.215 & 0.275 \\
\hline & LINE & 0.425 & 0.542 & 0.620 & 0.681 & 0.256 & 0.331 & 0.418 & 0.512 & 0.165 & 0.173 & 0.193 & 0.227 \\
\hline & HILL_Net & 0.550 & 0.622 & 0.685 & 0.741 & 0.313 & 0.396 & 0.495 & 0.624 & 0.287 & 0.272 & 0.156 & 0.141 \\
\hline & PCA & 0.691 & 0.780 & 0.821 & 0.855 & 0.510 & 0.612 & 0.671 & 0.696 & 0.591 & 0.599 & 0.605 & N.A. \\
\hline & Spectral & 0.714 & 0.788 & 0.838 & 0.867 & 0.695 & 0.767 & 0.810 & 0.843 & 0.610 & 0.626 & N.A. & N.A. \\
\hline & LCMF & 0.776 & 0.847 & 0.886 & 0.900 & 0.585 & 0.683 & 0.729 & 0.751 & 0.589 & 0.605 & 0.612 & N.A. \\
\hline & MultiSpec & 0.677 & 0.787 & 0.847 & 0.895 & 0.589 & 0.722 & 0.802 & 0.859 & 0.578 & 0.589 & N.A. & N.A. \\
\hline & HILL_Attri & 0.836 & 0.875 & 0.912 & 0.930 & 0.743 & 0.814 & 0.852 & 0.883 & 0.630 & 0.645 & 0.656 & 0.663 \\
\hline & HILL_Stream & 0.770 & 0.815 & 0.884 & 0.912 & 0.567 & 0.727 & 0.815 & 0.858 & 0.503 & 0.538 & 0.613 & 0.646 \\
\hline
\end{tabular}

Training set percentage indicates the percentage of nodes in training group that are used for embedding. \# Nodes for embedding denotes the total number of nodes in the test group and nodes in the training group that are used. HILL_Attri outperforms baselines with a 0.05 significance level.

Table 5. The Classification Performance of Different Scalable

Embedding Methods on Yelp with $d=100$, Where Baseline Methods PCA, LCMF, and MultiSpec are Infeasible

\begin{tabular}{lc|cccc}
\hline \multicolumn{2}{l|}{$\begin{array}{l}\text { Training set percentage } \\
\text { \# nodes for embedding }\end{array}$} & $10 \%$ & $25 \%$ & $50 \%$ & $100 \%$ \\
\multirow{2}{*}{ Micro- } & DeepWalk & 0.324 & 0.345 & 0.366 & 0.368 \\
average & LINE & 0.295 & 0.313 & 0.336 & 0.354 \\
& HILL_Attri & $\mathbf{0 . 6 9 8}$ & $\mathbf{0 . 7 0 9}$ & $\mathbf{0 . 7 1 1}$ & $\mathbf{0 . 7 1 4}$ \\
\hline \multirow{2}{*}{ Macro- } & DeepWalk & 0.239 & 0.254 & 0.266 & 0.260 \\
\multirow{2}{*}{ average } & LINE & 0.216 & 0.236 & 0.259 & 0.279 \\
& HILL_Attri & $\mathbf{0 . 6 4 9}$ & $\mathbf{0 . 6 5 9}$ & $\mathbf{0 . 6 6 0}$ & $\mathbf{0 . 6 6 5}$ \\
\hline
\end{tabular}

HILL_Attri outperforms baselines with a 0.05 significance level.

and MultiSpec. Second, to evaluate the performance of our framework on the second-order proximity learning, we compare HILL_Net with LINE, which learns a unified low-dimensional representation by concatenating the latent representations of first-order and second-order proximities straightforwardly. The classification results w.r.t. different training set percentages are shown in Table 4. From the results, we have two major observations as follows. First, in terms of node attribute learning, HILL_Attri consistently outperforms LCMF and MultiSpec w.r.t. all training set percentages. For instance, on Flickr, HILL_Attri achieves 18.2\% of improvements than LCMF when training set percentage is $100 \%$, which could be explained by the fact that latent features learned 

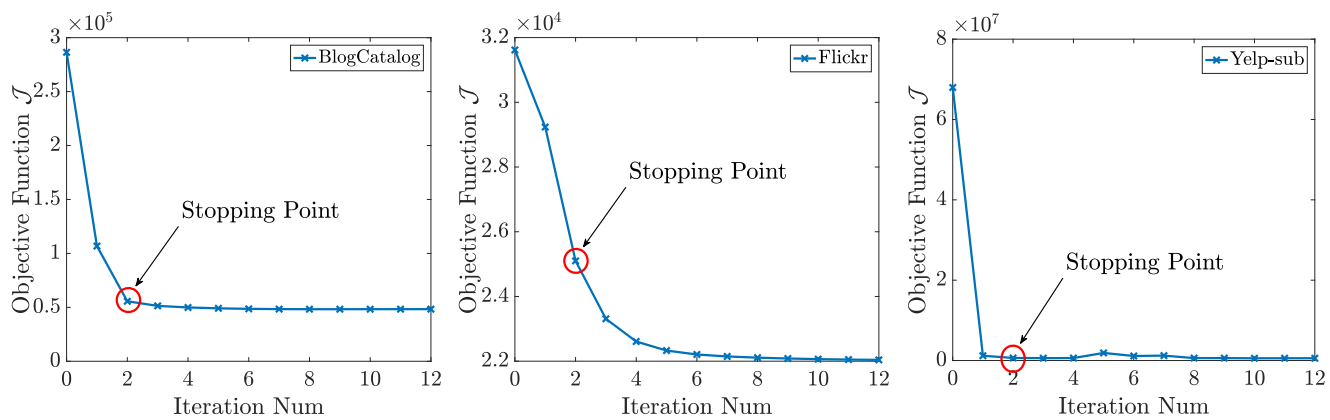

Fig. 3. The augmented Lagrangian $\mathcal{L}$ in HILL_Attri w.r.t. the iteration number on all the three datasets.

by decomposing the network matrix and node attribute information matrix are heterogeneous. It is challenging to combine them. We also find that MultiSpec might perform worse than PCA. For instance, on Yelp-sub, PCA achieves a gain of $1.0 \%$ over MultiSpec when training set percentage is $25 \%$. It is because the network information is too noisy. MultiSpec treats the networks and node attributes as two views, and incorporates them without considering their distinct data structures. Second, in terms of second-order proximity learning, HILL_Net consistently achieves better performance than LINE on BlogCatalog and Flickr. For instance, on BlogCatalog, HILL_Net achieves a gain of $9.0 \%$ over LINE, which could be explained by the fact that LINE combines the first-order and second-order proximities via simple concatenation, while HILL_Net jointly embeds them with a carefully designed structure.

One-tailed t-test results show that HILL_Attri is statistically significantly better (with p-value « $0.01)$ than all the baselines on all datasets. The p-value on BlogCatalog and Flickr are smaller than $9.5 \times 10^{-14}$ and $1.6 \times 10^{-12}$, respectively.

\subsection{Efficiency Evaluation}

To study the efficiency of the proposed framework, we compare HILL_Attri with the two heterogeneous information joint learning methods. Empirical results show that a near optimal solution of $\mathcal{J}$ is enough to guarantee $\mathbf{H}$ to be an informative embedding representation. Figure 3 shows the objective function $\mathcal{J}$ in HILL_Attri as a function of the iteration number on all the three datasets. As we can see, $\mathcal{J}$ decreases rapidly in the first two iterations. Therefore, in practice, only a few iterations are required for our framework HILL. If it is not specified, in the experiments, we stop the iteration at the stopping points shown in Figure 3. It should be noted that $\mathcal{J}$ might not decrease monotonically because of the non-differentiable points at $\mathbf{h}_{i}=\mathbf{z}_{j}^{t}$ in Equation (8).

The computation time in logarithmic scale as a function of the number of input nodes on the three datasets are shown in Figure 4. The blue, red, and yellow dash-dot curves show the performance of LCMF, MultiSpec, and HILL_Attri, respectively. From the results, we observe that HILL_Attri takes much less running time than LCMF and MultiSpec consistently. As the number of nodes increases, the performance difference raises up. The third subfigure shows that LCMF and MultiSpec have larger growth rates than HILL_Attri. They become infeasible when the number of nodes is greater than 49,802 and 29,881, respectively, due to the high computational cost and memory requirement of matrix factorization and eigen-decomposition. Furthermore, while the three methods are running within a single thread for a fair comparison, HILL_Attri could be implemented in multi-thread way as illustrated in Figure 2. This strategy could further improve the efficiency of HILL_Attri. We demonstrate the running time of HILL_Attri w.r.t. different numbers of workers $c$ on a dual-core processor in Table 6. As we can see, on all the three datasets, the 

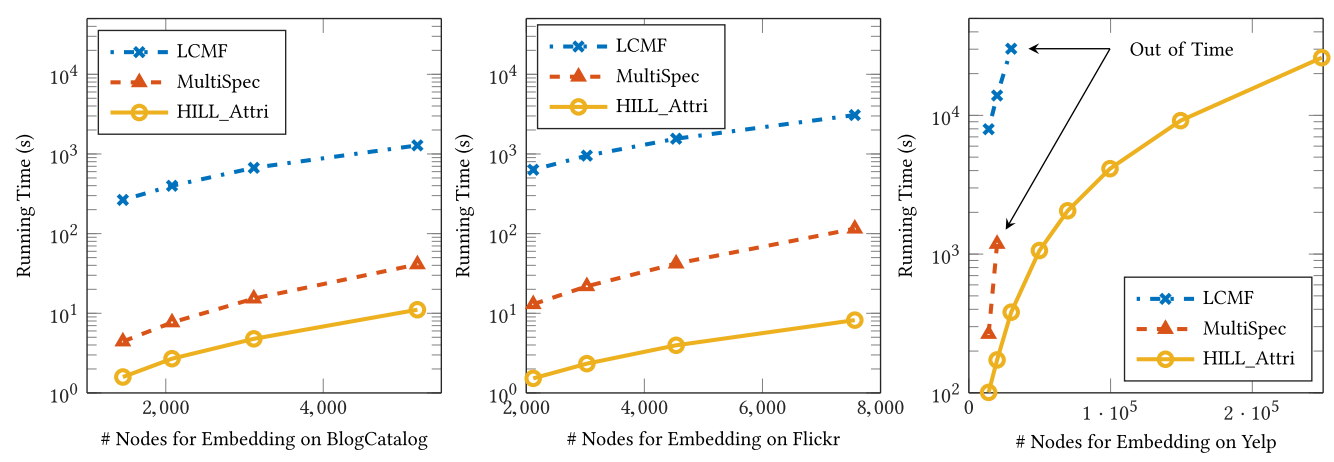

Fig. 4. Running time of LCMF, MultiSpec and HILL w.r.t. the number of input nodes within a single thread.

Table 6. The Running Time of HILL_Attri w.r.t. the Number of Workers $c$ on a Dual-core Processor

\begin{tabular}{l|ccc}
\hline & BlogCatalog (sec) & Flickr (sec) & Yelp-sub (sec) \\
\hline$c=1$ & 26.301 & 33.751 & 1065.033 \\
$c=2$ & $14.233(-45.9 \%)$ & $17.510(-48.1 \%)$ & $581.544(-45.4 \%)$ \\
\hline
\end{tabular}
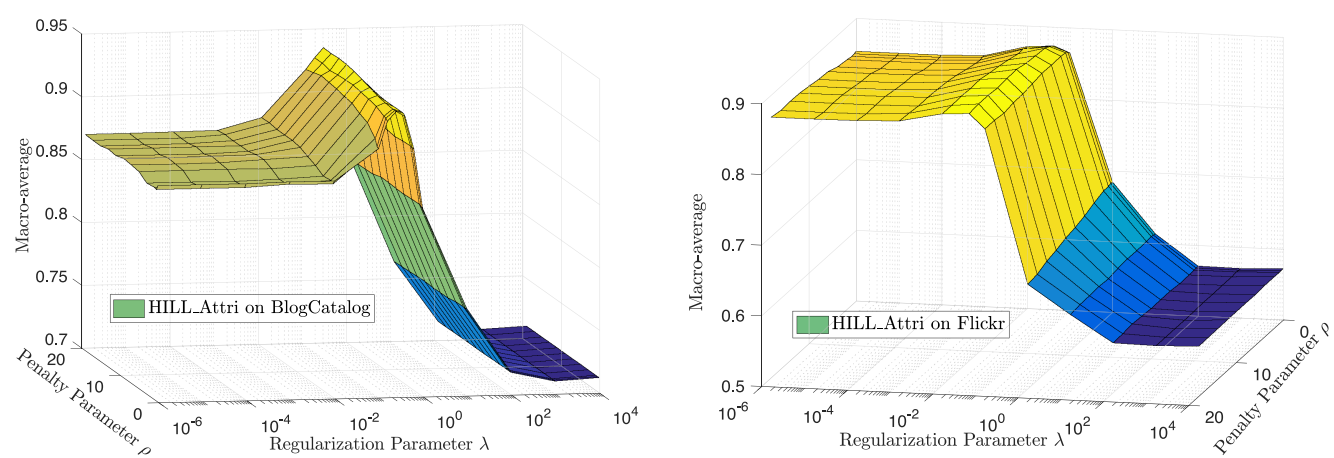

Fig. 5. Impacts of regularization parameter $\lambda$ and penalty parameter $\rho$ on the proposed framework HILL.

computation time of HILL_Attri is reduced by almost 50\% when $c$ is increased from 1 to 2 . When $c=1$, the running time on BlogCatalog and Flickr in Table 6 is larger than the one in Figure 4. It is because it takes extra time for the multi-thread version of HILL to set up the coordinator and workers. When $n$ is large enough, e.g., on Yelp-sub, the running time with $c=1$ in Table 6 is the same as the one in Figure 4. In summary, all these observations demonstrate the efficiency and scalability of HILL.

\subsection{Parameter Analysis}

We now answer the third proposed question, i.e., what are the impacts of parameters $\lambda$, $\rho$, and $d$. As discussed in Section 4.3, regularization parameter $\lambda$ in HILL balances the contributions of network information and heterogeneous information proximity. Penalty parameter $\rho$ determines the amount of penalty from the linear constraint $\mathbf{H}=\mathrm{Z}$. To investigate the impacts of $\lambda$ and $\rho$, we vary $\lambda$ from $10^{-6}$ to $10^{4}$ and $\rho$ from 0.1 to 20 . Figure 5 shows the performance in terms of 


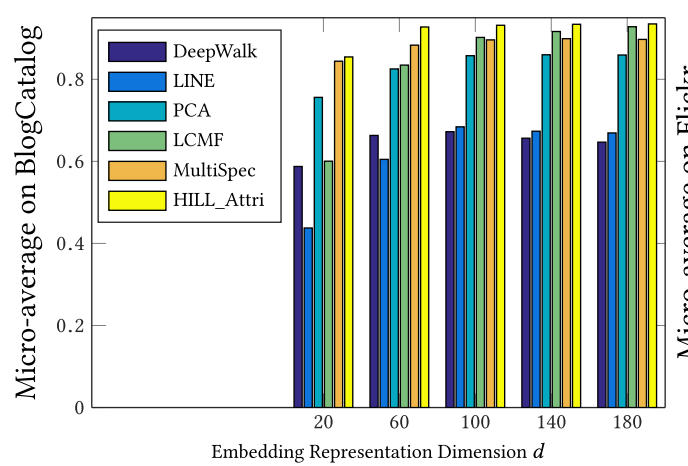

(a) BlogCatalog

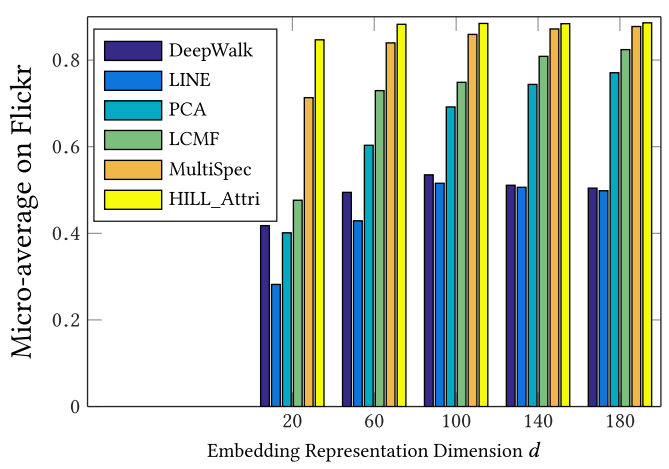

(b) Flickr

Fig. 6. The performance of all methods on BlogCatalog and Flickr w.r.t. different embedding dimension $d$.

Macro-average as a function of $\lambda$ and $\rho$ on BlogCatalog and Flickr. We omit the results on Yelp since they are similar.

From Figure 5, we have two observations. First, when $\lambda$ is close to 0 , network information has no impact as if all nodes are isolated. As $\lambda$ increases, HILL starts to cluster nodes according to the topological structure, so the performance keeps improving. As shown in Figure 5, performance on BlogCatalog and Flickr achieves optimal when $\lambda$ is close to 0.1 . The performance decreases when $\lambda$ is too large, since large $\lambda$ tends to drive all nodes to have the same vector representation. Second, the penalty parameter $\rho$ has a limited impact on the performance of our framework HILL.

To study the impact of embedding dimension $d$, we vary it from 20 to 180 on BlogCatalog and Flickr. The classification performance of all methods in terms of Micro-average w.r.t. $d$ is shown in Figure 6. We omit the results on Yelp since they are similar. From the results, we observe that conclusions made above hold undeviatingly as $d$ varies. DeepWalk and LINE are always inferior to HILL_Attri and the two heterogeneous information joint learning methods. HILL_Attri consistently outperforms all baselines. By increasing $d$, the performance of all methods first increases and then keeps stable. This shows that the learned low-dimensional representations perform well in capturing most of the meaningful information.

\subsection{Effectiveness Evaluation on Streaming Networks}

Our framework could also be applied to streaming networks. In the experiments, we make the nodes in the test group come one by one, and denote HILL on such streaming attributed network embedding as HILL_Stream. Its performance regards to different training set percentages is shown in Table 4. From the results, we observe that HILL_Stream achieves a comparable performance with the batch mode, i.e., HILL_Attri, on all the three datasets. For example, on Flickr, HILL_Stream performs only $2.8 \%$ worse than HILL_Attri when the training set percentage is $100 \%$. The performance of HILL_Stream on Yelp-sub is worse than PCA since HILL relies on the topological structure to infer the vector representations of new nodes, while the network information of Yelp-sub is noisy.

\section{RELATED WORK}

Our work is related to three research topics, i.e., large-scale network embedding, attributed network embedding, and network lasso, with detailed descriptions as follows.

First, large-scale network embedding has become an efficient tool to deal with real-world networks. The main question is how to efficiently learn low-dimensional representations for all 
vertices in a large network, such that the original geometrical information is recoverable. Efforts have been devoted from various aspects [11, 40,44]. Tang and Liu [45] presented an edge-centric clustering scheme to facilitate the learning efficiency and alleviate the memory demand. Ahmed et al. [1] advanced a distributed matrix factorization algorithm to decompose large-scale graphs based on stochastic gradient descent. Tang et al. [44] improved the efficiency of stochastic gradient descent via an edge-sampling algorithm. The basic idea is to unfold a weighted edge into many binary edges by sampling each edge with a probability proportional to the weight. Ou et al. [36] designed a Jacobi-Davidson type algorithm to approximate and accelerate the singular value decomposition in the high-order proximity embedding. Wang et al. [52] involved a deep structure to embed both first- and second-order proximities of nodes. Grover and Leskovec [11] involved language modeling techniques to make the network embedding scalable. The basic idea is to conduct truncated random walks on a graph and analyze them as sentences. Two key parameters are defined to bias the walks toward broader or deeper space. Dong et al. [7] further advanced this idea to embed networks with heterogeneous types of nodes, and designed a novel heterogeneous skip-gram model to jointly learn structural and semantic correlations. Yang et al. [55] proposed to accelerate the embedding by approximating the network proximity via a theoretical bound. Most recently, a series of deep learning based embedding models [50,52] have been explored. The focus of our article is scalable models, and a survey on network embedding could be found in [6].

Second, research has been done to analyze attributed networks in various domains. It becomes increasingly promising to advance the learning performance by jointly exploiting geometrical structure and node attributes [16, 17, 43]. Tsur and Rappoport [49] improved the prediction of the spread of ideas by taking advantage of both content and topological features. Due to the complexity of attributed networks, nodes' properties and dependencies cannot be fully explained via these models. Several algorithms $[29,58]$ have been proposed to exploit the potential of learning a more informative joint low-rank latent representation. Qi et al. [41] modeled the content information based on pairwise similarity and jointly mapped it along with context link into a semantic latent space by learning structural correlation between semantic concepts. Le and Lauw [27] advocated a probabilistic-based framework for the document network by finding a joint low-dimensional representation for network connectivity and textual content. Chang et al. [4] designed a deep learning method to map the rich linkage and content information into a latent space while capturing the similarities among cross-modal data. Yang et al. [54] proposed a coupled matrix factorizationbased method to handle networks with text information describing the nodes. Efforts also have been devoted to learning latent representations of networked instances by performing unsupervised feature selection [29, 30]. Kipf and Welling [23] introduced a variational graph autoencoder to learn a joint latent representation from an undirected graph and node features. They further improved the performance of deep models via a graph convolutional neural structure [24]. Huang et al. [18] and Pan et al. [38] explored the potential of incorporating labels into the attributed network embedding. Liu et al. [32] performed local anomaly detection based on attributed network embedding, and accelerated the embedding process via a parallel mini-batch stochastic gradient descent. Li et al. [28] explored the attributed network embedding in a dynamic environment, and proposed an online framework based on the matrix perturbation theory. Most recently, an interactive framework [19] has been proposed to learn and incorporate the meaningful but abstract experts' knowledge into the attributed network embedding. Attributed network analysis is different from multi-view learning [26]. The network structure is more than one angle of view. Its underlying properties are complex, including the connectivity, transitivity [36], first and higher order proximities [52], and so on.

Third, network lasso was formally defined by Hallac et al. [12] as a simultaneous clustering and optimization problem. The key idea is to utilize the $\ell_{2}$-norm distances of adjacent nodes 
as penalties and enforce nodes in the same cluster to have similar representations. The pioneer work of network lasso could be traced back to the fused lasso [15, 47] and convex clustering [39] problems. Both of them can be viewed as special cases of network lasso and have been well explored. Lindsten et al. [31] demonstrated the equivalence between convex clustering and a convexification of $k$-means clustering. They utilized the off-the-shelf convex programming software CVX to handle the regularization. This solver is powerful in solving general convex problems but quite inefficient. Thus, Hocking et al. [14] introduced several efficient algorithms for convex clustering with three commonly used norms, $\ell_{1}, \ell_{2}$, and $\ell_{\infty}$. Recently, Chi and Lange [5] exploited the possibility of finding regularization paths of arbitrary norms by using ADMM and alternating minimization algorithm. These methods could only deal with thousands of nodes, and convex clustering often requires to include distances between every pair of nodes as penalties. We propose to use network lasso and symmetric matrix factorization jointly to perform the heterogeneous information learning. It is novel and non-trivial since the framework is required to be both separable and effective. Efforts also have been devoted to proving the monotonicity of the proposed updated rules.

\section{CONCLUSION AND FUTURE WORK}

In this article, we propose a general and efficient embedding framework HILL to handle the heterogeneous information learning in real-world networks at scale. The proposed framework could effectively model an arbitrary type of heterogeneous information by incorporating its node proximity into network embedding in a distributed manner. Specifically, we learn a low-dimensional representation based on the decomposition of the heterogeneous information affinity matrix and the embedding difference between connected nodes. A distributed optimization algorithm is developed to decompose the complex problem into many sub-problems of low complexity, which could be solved by sub-workers in parallel. We demonstrate the generalizability of HILL with three real-world applications, including attributed network embedding, second-order proximity learning, and link directionality modeling. A variation of HILL to handle heterogeneous information learning in streaming networks is also proposed. Experiments on the three real-world attributed networks demonstrate the effectiveness and efficiency of HILL. Our future work in this area will focus on investigating several open questions as follows. (1) How can we embed large-scale networks with dynamic topological structure and dynamic heterogeneous information? (2) How can we extend the proposed algorithms to a semi-supervised framework, since in many real-world information networks, partial labels are often available?

\section{REFERENCES}

[1] Amr Ahmed, Nino Shervashidze, Shravan Narayanamurthy, Vanja Josifovski, and Alexander J. Smola. 2013. Distributed large-scale natural graph factorization. In International World Wide Web Conference. 37-48.

[2] Lars Backstrom and Jure Leskovec. 2011. Supervised random walks: Predicting and recommending links in social networks. In ACM International Conference on Web Search and Data Mining. 635-644.

[3] Stephen Boyd, Neal Parikh, Eric Chu, Borja Peleato, and Jonathan Eckstein. 2011. Distributed optimization and statistical learning via the alternating direction method of multipliers. Foundations and Trends ${ }^{\circledR}$ in Machine Learning 3 , 1 (2011), 1-122.

[4] Shiyu Chang, Wei Han, Jiliang Tang, Guo-Jun Qi, Charu C. Aggarwal, and Thomas S. Huang. 2015. Heterogeneous network embedding via deep architectures. In ACM SIGKDD International Conference on Knowledge Discovery and Data Mining. 119-128.

[5] Eric C. Chi and Kenneth Lange. 2015. Splitting methods for convex clustering. Journal of Computational and Graphical Statistics 24, 4 (2015), 994-1013.

[6] Peng Cui, Xiao Wang, Jian Pei, and Wenwu Zhu. 2017. A survey on network embedding. In IEEE Transactions on Knowledge and Data Engineering (2017).

[7] Yuxiao Dong, Nitesh V. Chawla, and Ananthram Swami. 2017. metapath2vec: Scalable representation learning for heterogeneous networks. In ACM SIGKDD International Conference on Knowledge Discovery and Data Mining. 135144. 
[8] Ahmed El Alaoui, Xiang Cheng, Aaditya Ramdas, Martin J. Wainwright, and Michael I. Jordan. 2016. Asymptotic behavior of $\ell_{\mathrm{p}}$-based laplacian regularization in semi-supervised learning. Journal of Machine Learning Research 49 (2016), 1-28.

[9] Ron Eyal, Avi Rosenfeld, Sigal Sina, and Sarit Kraus. 2014. Predicting and identifying missing node information in social networks. ACM Transactions On Knowledge Discovery From Data 8, 3 (2014), 1-35.

[10] Xiang Gao and Shu-Zhong Zhang. 2017. First-order algorithms for convex optimization with nonseparable objective and coupled constraints. Journal of the Operations Research Society of China 5, 2 (2017), 131-159.

[11] Aditya Grover and Jure Leskovec. 2016. node2vec: Scalable feature learning for networks. In ACM SIGKDD International Conference on Knowledge Discovery and Data Mining. 855-864.

[12] David Hallac, Jure Leskovec, and Stephen Boyd. 2015. Network lasso: Clustering and optimization in large graphs. In ACM SIGKDD International Conference on Knowledge Discovery and Data Mining. 387-396.

[13] Magnus R. Hestenes. 1969. Multiplier and gradient methods. Fournal of Optimization Theory and Applications 4, 5 (1969), 303-320.

[14] Toby Dylan Hocking, Armand Joulin, Francis Bach, and Jean-Philippe Vert. 2011. Clusterpath: An algorithm for clustering using convex fusion penalties. International Conference on Machine Learning (2011), 745-752.

[15] Holger Hoefling. 2010. A path algorithm for the fused lasso signal approximator. fournal of Computational and Graphical Statistics 19, 4 (2010), 984-1006.

[16] Xia Hu, Lei Tang, Jiliang Tang, and Huan Liu. 2013. Exploiting social relations for sentiment analysis in microblogging. In ACM International Conference on Web Search and Data Mining. 537-546.

[17] Jin Huang, Feiping Nie, Heng Huang, Yi-Cheng Tu, and Yu Lei. 2013. Social trust prediction using heterogeneous networks. ACM Transactions On Knowledge Discovery From Data 7, 4 (2013), 1-21.

[18] Xiao Huang, Jundong Li, and Xia Hu. 2017. Label informed attributed network embedding. In ACM International Conference on Web Search and Data Mining. 731-739.

[19] Xiao Huang, Qingquan Song, Jundong Li, and Xia Hu. 2018. Exploring expert cognition for attributed network embedding. In ACM International Conference on Web Search and Data Mining. 270-278.

[20] Zepeng Huo, Xiao Huang, and Xia Hu. 2018. Link prediction with personalized social influence. In AAAI Conference on Artificial Intelligence.

[21] Ling Jian, Jundong Li, Kai Shu, and Huan Liu. 2016. Multi-label informed feature selection. In International foint Conference on Artificial Intelligence. 1627-1633.

[22] Ian T. Jolliffe. 1986. Principal component analysis and factor analysis. In Principal Component Analysis. Springer, 115-128.

[23] Thomas N. Kipf and Max Welling. 2016. Variational graph auto-encoders. In NIPS Workshop on Bayesian Deep Learning.

[24] Thomas N. Kipf and Max Welling. 2017. Semi-supervised classification with graph convolutional networks. In International Conference on Learning Representations.

[25] Da Kuang, Chris Ding, and Haesun Park. 2012. Symmetric nonnegative matrix factorization for graph clustering. In SIAM International Conference on Data Mining. 106-117.

[26] Abhishek Kumar, Piyush Rai, and Hal Daume. 2011. Co-regularized multi-view spectral clustering. In Conference on Neural Information Processing Systems. 1413-1421.

[27] Tuan M. V. Le and Hady W. Lauw. 2014. Probabilistic latent document network embedding. In IEEE International Conference on Data Mining. 270-279.

[28] Jundong Li, Harsh Dani, Xia Hu, Jiliang Tang, Yi Chang, and Huan Liu. 2017. Attributed network embedding for learning in a dynamic environment. In ACM International Conference on Information and Knowledge Management.

[29] Jundong Li, Xia Hu, Jiliang Tang, and Huan Liu. 2015. Unsupervised streaming feature selection in social media. In ACM International Conference on Information and Knowledge Management. 1041-1050.

[30] Jundong Li, Xia Hu, Liang Wu, and Huan Liu. 2016. Robust unsupervised feature selection on networked data. In SIAM International Conference on Data Mining. 387-395.

[31] Fredrik Lindsten, Henrik Ohlsson, and Lennart Ljung. 2011. Just Relax and Come Clustering!: A Convexification of k-means Clustering. Linköping University Electronic Press (2011).

[32] Ninghao Liu, Xiao Huang, and Xia Hu. 2017. Accelerated local anomaly detection via resolving attributed networks. In International foint Conference on Artificial Intelligence. 2337-2343.

[33] Peter V. Marsden. 1988. Homogeneity in confiding relations. Social Networks 10, 1 (1988), 57-76.

[34] Miller McPherson, Lynn Smith-Lovin, and James M. Cook. 2001. Birds of a feather: Homophily in social networks. Annual Review of Sociology 27, 1 (2001), 415-444.

[35] Hariharan Narayanan, Mikhail Belkin, and Partha Niyogi. 2006. On the relation between low density separation, spectral clustering and graph cuts. In Conference on Neural Information Processing Systems. 1025-1032. 
[36] Mingdong Ou, Peng Cui, Jian Pei, and Wenwu Zhu. 2016. Asymmetric transitivity preserving graph embedding. In ACM SIGKDD International Conference on Knowledge Discovery and Data Mining. 1105-1114.

[37] Mingdong Ou, Peng Cui, Fei Wang, Jun Wang, Wenwu Zhu, and Shiqiang Yang. 2013. Comparing apples to oranges: A scalable solution with heterogeneous hashing. In ACM SIGKDD International Conference on Knowledge Discovery and Data Mining. 230-238.

[38] Shirui Pan, Jia Wu, Xingquan Zhu, Chengqi Zhang, and Yang Wang. 2016. Tri-party deep network representation. In International foint Conference on Artificial Intelligence. 1895-1901.

[39] Kristiaan Pelckmans, Joseph De Brabanter, J. A. K. Suykens, and B. De Moor. 2005. Convex clustering shrinkage. In PASCAL Workshop on Statistics and Optimization of Clustering Workshop.

[40] Bryan Perozzi, Rami Al-Rfou, and Steven Skiena. 2014. DeepWalk: Online learning of social representations. In ACM SIGKDD International Conference on Knowledge Discovery and Data Mining. 701-710.

[41] Guo-Jun Qi, Charu Aggarwal, Qi Tian, Heng Ji, and Thomas S. Huang. 2012. Exploring context and content links in social media: A latent space method. IEEE Transactions on Pattern Analysis and Machine Intelligence 34, 5 (2012), 850-862.

[42] Jiliang Tang, Charu Aggarwal, and Huan Liu. 2016. Node classification in signed social networks. In SIAM International Conference on Data Mining. 54-62.

[43] Jiliang Tang, Huiji Gao, Xia Hu, and Huan Liu. 2013. Exploiting homophily effect for trust prediction. In ACM International Conference on Web Search and Data Mining. 53-62.

[44] Jian Tang, Meng Qu, Mingzhe Wang, Ming Zhang, Jun Yan, and Qiaozhu Mei. 2015. LINE: Large-scale information network embedding. In International World Wide Web Conference. 1067-1077.

[45] Lei Tang and Huan Liu. 2009. Scalable learning of collective behavior based on sparse social dimensions. In ACM International Conference on Information and Knowledge Management. 1107-1116.

[46] Robert Tibshirani. 1996. Regression shrinkage and selection via the lasso. Journal of the Royal Statistical Society: Series B (1996), 267-288.

[47] Robert Tibshirani, Michael Saunders, Saharon Rosset, Ji Zhu, and Keith Knight. 2005. Sparsity and smoothness via the fused lasso. Fournal of the Royal Statistical Society: Series B 67, 1 (2005), 91-108.

[48] Koji Tsuda, Hyunjung Shin, and Bernhard Schölkopf. 2005. Fast protein classification with multiple networks. Bioinformatics 21, suppl_2 (2005), ii59-ii65.

[49] Oren Tsur and Ari Rappoport. 2012. What's in a hashtag? Content based prediction of the spread of ideas in microblogging communities. In ACM International Conference on Web Search and Data Mining. 643-652.

[50] Ke Tu, Peng Cui, Xiao Wang, Fei Wang, and Wenwu Zhu. 2018. Structural deep embedding for hyper-networks. In AAAI Conference on Artificial Intelligence.

[51] Ulrike von Luxburg. 2007. A tutorial on spectral clustering. Statistics and Computing 17, 4 (2007), 395-416.

[52] Daixin Wang, Peng Cui, and Wenwu Zhu. 2016. Structural deep network embedding. In ACM SIGKDD International Conference on Knowledge Discovery and Data Mining. 1225-1234.

[53] Xiao Wang, Peng Cui, Jing Wang, Jian Pei, Wenwu Zhu, and Shiqiang Yang. 2017. Community preserving network embedding. In AAAI Conference on Artificial Intelligence. 203-209.

[54] Cheng Yang, Zhiyuan Liu, Deli Zhao, Maosong Sun, and Edward Y. Chang. 2015. Network representation learning with rich text information. In International foint Conference on Artificial Intelligence. 2111-2117.

[55] Cheng Yang, Maosong Sun, Zhiyuan Liu, and Cunchao Tu. 2017. Fast network embedding enhancement via high order proximity approximation. In International foint Conference on Artificial Intelligence. 19-25.

[56] Guo Zhang, Ying Ding, and Staša Milojević. 2013. Citation content analysis (CCA): A framework for syntactic and semantic analysis of citation content. Journal of the Association for Information Science and Technology 64, 7 (2013), 1490-1503.

[57] Jing Zhang, Jie Tang, Juanzi Li, Yang Liu, and Chunxiao Xing. 2015. Who influenced you? Predicting retweet via social influence locality. ACM Transactions On Knowledge Discovery From Data 9, 3 (2015), 1-26.

[58] Shenghuo Zhu, Kai Yu, Yun Chi, and Yihong Gong. 2007. Combining content and link for classification using matrix factorization. In Special Interest Group on Information Retrieval. 487-494.

Received December 2017; revised May 2018; accepted July 2018 